BNL-63953

AD/RHIC-140

Informal Report

Some Calculations for the RHIC Kicker

J. Claus

December 1996

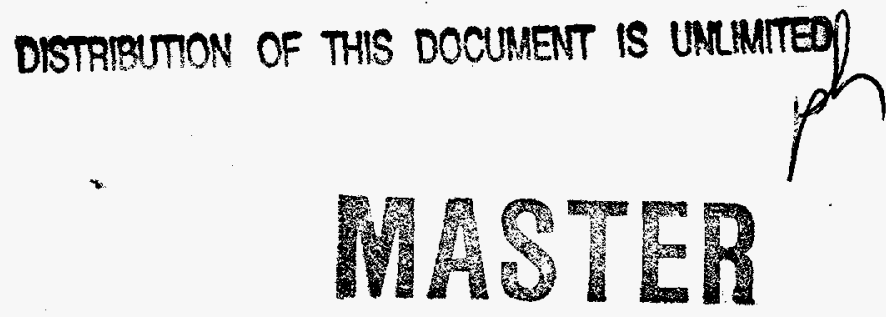

R H I P R O J E T

Brookhaven National Laboratory

Associated Universities, Inc.

Upton, NY 11973

Under Contract No. DE-AC02-76CH00016 with the

UNITED STATES DEPARTMENT OF ENERGY 


\section{DISCLAIMER}

This report was prepared as an account of work sponsored by an agency of the United States Government. Neither the United States Government nor any agency thereof, nor any of their employees, make any warranty, express or implied, or assumes any legal liability or responsibility for the accuracy, completeness, or usefulness of any information, apparatus, product, or process disclosed, or represents that its use would not infringe privately owned rights. Reference herein to any specific commercial product, process, or service by trade name, trademark, manufacturer, or otherwise does not necessarily constitute or imply its endorsement, recommendation, or favoring by the United States Government or any agency thereof. The views and opinions of authors expressed herein do not necessarily state or reflect those of the United States Government or any agency thereof. 


\section{DISCLAMMER}

Portions of this document may be illegible in electronic image products. Images are produced from the best available original document. 


\title{
Some Calculations for the RHIC Injection Kicker
}

\author{
J. Claus
}

September 1996

\section{Introduction}

The bunches that arrive from the AGS are put on to RHIC's median plane by a string of four injection kickers in each ring. There are four short kickers rather than one long one in order to keep the kicker filling time acceptable, filling time being defined as the amount of time needed for increasing the deflecting field in the kicker from zero to its nominal value. During the filling time process the energy stored in the deflecting field is moved from outside the kicker to its aperture; since energy can only be displaced with finite velocity the filling time is non-zero for kickers of non-zero length, and tends to increase with increasing length. It is one of the more important parameters of the kicker because it sets a lower limit to the time interval between the last of the already circulating bunches and the newly injected one, and thus an upper limit to the total number of bunches that can be injected. RF gymnastics can be used to pack the bunches tighter than is indicated by this limit, but such gymnastics required radial aperture beyond what would be required otherwise, as well as time, and probably special hardware. Minimization of the kicker's stored energy requires minimization of its aperture, it presents therefore a major aperture restriction. Unless it is placed at a point where the dispersion is negligible its aperture would have to be increased in order to provide the radial space needed for the gymnastics. Both the amount of extra space needed and the rate of longitudinal displacement increase with the maximum deviation in energy of the bunch to be displaced from the nominal value, thus taking more time for the exercise reduces the aperture requirements. This time is measured in terms of synchrotron periods and is not small. It adds directly to the filling time of each ring and decreases therefore the time-average luminosity. Evidently the maximation of the time-average luminosity is a complex issue in which the kicker filling time is a major parameter. 
The pulses that energize RHIC's injection kickers are made by Blumlein circuits that are located at such a distance from the kicker proper that the tail end of a pulse leaves the Blumlein before its front reaches the kicker. It follows that during the pulse the Blumlein is loaded with the characteristic impedance of the transmission line between it and the kicker, regardless of the electrical characteristics of the kicker, while the kicker receiving the pulse sees a source impedance equal to the line's characteristic impedance, regardless of the properties of the Blumlein. The net filling time is then composed of the risetime of the pulse delivered by the Blumlein and the response of the kicker to a stepfunction.

In first approximation the kicker may be seen as a piece of modified transmission line. It consists of either one or two inner conductors inside a tubelike outer conductor, the cross-sectional dimensions are invariant along its length (thus inner and outer conductors are parallel). However, in a transmission line the medium between the conductors line tends to be uniform in properties and uniformly distributed over the available space, this makes the EM behavior fairly simple: the characteristic impedance and velocity of wave propagation can be written in terms of inductance $\mathcal{L}$ and capacitance $\mathcal{C}$ per unit length, $\mathcal{Z}=(\mathcal{L} / \mathcal{C})^{1 / 2}, v=(\mathcal{L C})^{-1 / 2}$, and tend to be independent of frequency. In kickers the aperture reserved for the beam must remain empty, thus uniformity of properties and distribution can only be had in kickers with vacuum as medium between the conductors. Such kickers are inefficient because in them only a fraction of the field energy is stored within the beam aperture, the rest is uselessly hidden in regions that are inaccessible for the particles. Filling those regions with a material of high permeability, e.g., a high $\mu$ ferrite, improves the efficiency because then nearly all the magnetic energy is stored in the aperture. The presence of the ferrite increases $\mathcal{L}$ and $\mathcal{C}$, thus the magnetic inductance $B(\propto$ $\mathcal{L} / I)$ per unit excitation current, and the characteristic impedance $\mathcal{Z}$, it reduces the velocity of wave propagation $v$ and increases therefore the filling time. $\mathcal{C}$ tends to increase also, because $\epsilon_{r}>1$ for ferrites. Since the beam aperture must remain vacuum the distribution of the medium is non-uniform. EM-field calculations become much more complicated, and the system becomes dispersive if the results of calculations done for a model with a very simple geometry are any indication. The validity of the usual transmission line expressions becomes therefore questionable. We shall disregard this remark for the moment and assume in what follows that the transmission line formula remain valid, in particular $\mathcal{Z}=(\mathcal{L} / \mathcal{C})^{1 / 2}$ 
and $v=1 /(\mathcal{L C})^{1 / 2}=\mathcal{Z} / \mathcal{L}$, where $\mathcal{L}$ and $\mathcal{C}$ are estimated from the actual cross-sectional dimensions of the kicker; they are certainly valid for sufficiently low frequencies.

The characteristic impedances of ferrite loaded kickers tend to be high, which leads to undesirably high excitation voltages. The inductance of a kicker with a square aperture ( $w \cong g, w=$ aperture with, $g=$ gap height) is $\mathcal{L} \cong 1.26 \mu \mathrm{H} / \mathrm{m}, \mathcal{Z} \cong 25 \Omega$ would require $\mathcal{C} \cong 2 \mathrm{nF} / \mathrm{m}$, but an excitation current of $1.5 \mathrm{kA}$ for $B \cong 0.04 \mathrm{~T}$ in a $48 \mathrm{~mm}$ gap still requires a voltage of $1.5 \times 25=37.5 \mathrm{kV}$. It may also be noted that $v \cong 2.10^{7} \mathrm{n} / \mathrm{sec}=$ $0.066 \mathrm{c}$. Because it is difficult to make the 'natural' $\mathcal{C}$ sufficiently large lumped capacitors are often added periodically along the length of a kicker in order to reduce the characteristic impedance to a desired level. This approximation of a large $\mathcal{C}$ works well for low frequencies but is expensive because the originally simple magnet design becomes much more complicated. SLAC's Cassel restored mechanical simplicity in an elegant design in which the kicker is longitudinally subdivided in alternating inductive and capacitive sections. These sections have identical cross-sectional geometries, but the regions that are filled with ferrite in the inductive sections are filled with a high permittivity ceramic in the capacitive ones, see Fig. 1. The design of RHIC's injection kickers follows this approach with the difference that only the ferrite in the backleg, a region of high electric field, is replaced by the high permittivity ceramic, while the ferrite in the poleplates remains, see Fig. 2. ${ }^{1}$ The capacitive sections replace the lumped capacitors of the original arrangement. The longitudinal modulation of the kicker structure has serious electrical consequences: it introduces stopbands, and more generally, a strong frequency dependence in the originally flat curves of all parameters of interest. The correspondence between the continuous and longitudinally modulated structures holds only for frequencies well below the lower edge of the first stopband. The charging wave becomes distorted on its passage through the kicker and the risetime increases with decreasing lower edge frequency. The frequencies of the band edges depend upon the lengths of the inductive and capacitive sections: the shorter the sections, the higher the frequencies. It is therefore desirable to subdivide the kicker in as many short sections as practicable. Cassel's design is particularly suitable

1 Figs. 1 and 2 were copied from AD/RHIC/RD-67 (H. Hahn, E.B. Forsyth, Transmission Line Analysis of Dielectric Loaded Ferrite Kicker) and AD/RHIC/RD-105 (H. Hahn, A. Ratti, The Coupling Impedance of the RHIC Injection Kicker System). 
for short sections since it does not have to provide space for high voltage connections to lumped capacitors. The frequency dependence of the various electrical parameters decreases when the lengths of the sections approach their transverse dimensions and return to flatness when they are much smaller, because end effects (stray fields), negligible when the sections are long, become relatively stronger and finally dominate. The modulation amplitudes of the fields decrease relative to their average values and become negligible. When that stage is reached the kicker behaves as a longitudinally uniform structure and the stopbands disappear. The effective permeability and permittivity in that structure are now the weighted average of their values in the magnetic and capacitive sections at the corresponding transverse locations:

$$
\begin{aligned}
\epsilon_{\mathrm{eff}} & =\epsilon_{\mathrm{fer}} \ell_{\mathrm{fer}}+\epsilon_{\mathrm{cer}} \ell_{\mathrm{cer}} \\
\mu_{\mathrm{eff}} & =\mu_{\mathrm{fer}} \ell_{\mathrm{fer}}+\mu_{\mathrm{cer}} \ell_{\mathrm{cer}}
\end{aligned}
$$

where $\ell_{\text {fer }}$ and $\ell_{\text {cer }}, \ell_{\text {fer }}+\ell_{\text {cer }}=1$, are the fractions of the total length that are occupied by ferrite and high permittivity ceramic respectively. The characteristic impedance is independent of frequency and its values can be controlled by appropriate choice of the $\ell_{\text {fer }}<$ 1 , provided that a ceramic with a sufficiently high $\epsilon_{r}$ is available. Analytic determination of the section lengths required to establish this condition requires solution of Maxwell's equations, an educated guess suggests that they should be small fractions, e.g., $\cong 0.1$ of the smallest aperture dimension. They can also be established experimentally by checking for resonant behavior and/or measurement of the characteristic impedance of a short prototype kicker which contains several magnetic and capacitive sections; the sections are too long if there is a marked frequency dependence. The educated guess implies that RHIC's injection kickers would have a frequency independent characteristic impedance $\mathcal{Z} \cong 25 \Omega$ if their backlegs consisted of ferrite strips with $\mathrm{h} \times \mathrm{w} \times \mathrm{t} \cong 76.2 \times 13.9 \times 5.86 \mathrm{~mm}^{3}$ and ceramic strips with $\mathrm{h} \times \mathrm{w} \times \mathrm{t} \cong 76.2 \times 13.9 \times 4.14 \mathrm{~mm}^{3}$. A kicker length of $1 \mathrm{~m}$ implies 100 ferrite strips and 100 ceramic strips. Some parameters of such a kicker are given in Table I. 
Field aperture $(w \times g)$

$46.43 \times 48.41$

$\mathrm{mm}^{2}$

Characteristic impedance

$\mathcal{Z} \quad 25 \Omega$

Wave velocity

v $\quad 2.07 \times 10^{7}$

$\mathrm{m} / \mathrm{sec}=0.069 \mathrm{c}$

Fillingtime(chargingwave stepfunction) $\quad t$

Deflection $(\mathrm{B} \rho=100 \mathrm{Tm})$

$1 / 2.07 \times 107=48.2 \quad$ nsec

Magnetic component of deflecting field

$\Delta \phi$

$1.9 / 4=0.475$

mrad

Electric component of deflecting field

$B_{\text {defl }}$

0.0444

$\mathrm{T}$

Excitation current

$E_{\text {defl }}$

0.921

$\mathrm{MV} / \mathrm{m}$

Excitation voltage

I

1.71

$\mathrm{KA}$

Magnetic induction in backleg ferrite

$\mathrm{U}$

42.77

KV

$B_{\text {backleg }} 0.0253$

$\mathrm{T}$

Electric field in backleg ceramic

$E_{\text {backleg }} 3$.

$\mathrm{MV} / \mathrm{m}$

Since the physical charging wave cannot be a stepfunction the net filling time is the sum of its risetime and the kicker's filling time; a net filling time of $80 \mathrm{nsec}^{2}$ requires therefore a risetime of the charging wave of about $\$ 30 \mathrm{nsec}$. The present design for RHIC's injection kickers call for longitudinal and capacitive sections are of the same order as the aperture, not much larger nor much smaller. This makes accurate analytical prediction of their behavior very difficult. In order to gain at least some qualitative insight of that behavior we performed calculations which are based on the actual dimensions of the kickers but which neglect the end effects of the individual sections. The effects of the sectionalisation are therefore exaggerated relative to reality in the results.

2 See BNL-61172: V. Mane, et. al., RHIC Injection Kicker Impedance. 


\section{Calculations}

The problem was addressed analytically as well as numerically. The goal of the analytical approach is to obtain the kicker's response to a stepfunction via Laplace transforms; however, the final step of transforming the results back to the time domain proved too cumbersome to tackle. It did yield a description of the behavior as function of frequency as a consequence of the close relation between Laplace and Fourier transforms.

The numerical approach presents problems also. A direct simulation in time ${ }^{3}$ presents little difficulty but was abandoned when it appeared that simulation during sufficiently large time intervals of kickers of this complexity requires unacceptably large amounts of computer memory and processing time. This was the reasons for switching to Fourier methods. However, because the numerical resolution of any digital computer is finite, the true Fourier transform of the stepfunction must be replaced by a discrete Fourier transform. Such transforms can inherently only treat periodic functions, describing them with a finite number of harmonics of a fundamental period. The highest harmonic has necessarily a finite frequency, its choice determines the achievable resolution in time. Keeping it fixed while increasing the number of harmonics increases the fundamental period, increases the density of harmonics and decreases their amplitudes. E.g., choosing the maximum frequency at $100 \mathrm{Mhz}$, in order to obtain a time resolution of the order of $5 \mathrm{nsec}$, i.e., well within, but not much smaller than, the bunch length of about $15 \mathrm{nsec}$, and working with 1024 harmonics puts the fundamental frequency and the distance between successive harmonics at about $100 \mathrm{kHz}$ and the fundamental period at about $10 \mu \mathrm{sec}$, i.e., slightly smaller than the $12 \mu \mathrm{sec}$ revolution period in the RHIC rings. It seems desirable, for reasons to be mentioned below, to increase the number of harmonics by a large factor, e.g., 128, but this is hardly possible because of the correspondingly increased demands on memory, file lengths, and processing time. The net result is that the stepfunction is

3 These simulations were done by means of / claus/chain1,f77 source code / claus/chain1.f, available on the RS6000 at AX61. 
replaced by a nearly square wave ${ }^{4}$, which one has to accept as a fairly, but not terribly, good approximation.

In both approaches the kicker is represented as a sequence of pieces of lossfree transmission line, each piece characterized by its inductance $\mathcal{L}$ and capacitance $\mathcal{C}$ per unit length or, equivalently, by its characteristic impedance $\mathcal{Z}=(\mathcal{L} / \mathcal{C})^{1 / 2}$, the velocity of wave propagation in it $v=(\mathcal{L C})^{-1 / 2}$, and by its length $\ell$. The transfer matrix $[h]$ for a piece is

$$
[h]=\left[\begin{array}{cc}
\cosh (\gamma \ell) & \mathcal{Z} \sinh (\gamma \ell) \\
\frac{\sinh (\gamma \ell)}{\mathcal{Z}} & \cosh (\gamma \ell)
\end{array}\right]
$$

where $\gamma=p(\mathcal{L C})^{1 / 2}$, with $p$ either the Laplace variable or $p=j \omega=j 2 \pi v, j=\sqrt{(-1)}$, with the $v$ the frequency. (1) is a solution of the familiar telegraph equations ${ }^{5}$ of conventional transmission line theory for a piece of lossless transmission line. Note that $\operatorname{Det}[h]=1$, a consequence of the absence of losses, and that the independent variable is $p \ell$ or $\omega \ell$, rather than $p$ or $\omega$. This implies that frequency dependencies scale as $1 / \ell$, time dependencies as $\ell$. For $(\gamma \ell) \ll 1$ the matrix may be approximated with

$$
[h]=\left[\begin{array}{cc}
1 & \rho \mathcal{L} \ell \\
p \mathcal{C} \ell & 1
\end{array}\right]
$$

which reduces the matrix for a series inductor $\mathcal{L} \ell$ is $\mathcal{C}$ is negligibly small and that for a parallel capacitor $\mathcal{C} \ell$ if $\mathcal{L}$ is negligibly small. The kicker is composed of only two kinds of transmission line sections: 15 magnetic sections alternate with 14 electric sections; the sections of a kind are nominally identical among each other but their lengths, inductances and capacitances may show small differences in practice. For analytical purposes we disregard the small differences and describe the structure as beginning with a half magnetic section, followed by 14 identical periods, each consisting of a capacitive section with a half magnetic section on either side, and ending with another half magnetic section. The matrix for the period may be written:

$$
\left[h_{p}\right]=\left[\begin{array}{cc} 
\pm \cosh (\psi) & \mathcal{Z}_{p} \sinh (\psi) \\
\frac{\sinh (\psi)}{\mathcal{Z}_{p}} & \pm \cosh (\psi)
\end{array}\right]
$$

where

$$
\cosh (\psi)=\left|\cosh \left(\gamma_{m} \ell_{m}\right) \cosh \left(\gamma_{e} \ell_{e}\right)+0.5\left(\frac{\mathcal{Z}_{m}}{\mathcal{Z}_{e}}+\frac{\mathcal{Z}_{e}}{\mathcal{Z}_{m}}\right) \sinh \left(\gamma_{m} \ell_{m}\right) \sinh \left(\gamma_{e} \ell_{e}\right)\right|
$$

${ }^{4}$ The true square wave is double valued at the instants of change of state, this nearly square wave is single valued at all points and changes states from one point to the next.

${ }^{5} \mathcal{L} \partial / \partial t=-\partial u / \partial z \quad \mathcal{C} \partial / \partial t=-\partial i / \partial z$. 


$$
\begin{aligned}
\mathcal{Z}_{p}= & \frac{\mathcal{Z}_{m}}{\sinh (\psi)}\left(\gamma_{m} \ell_{m}\right) \cosh \left(\gamma_{e} \ell_{e}\right)+0.5\left(\frac{\mathcal{Z}_{m}}{\mathcal{Z}_{e}}+\frac{\mathcal{Z}_{e}}{\mathcal{Z}_{m}}\right) \\
& \left.\cosh \left(\gamma_{m} \ell_{m}\right) \sinh \left(\gamma_{e} \ell_{e}\right)-0.5\left(\frac{\mathcal{Z}_{m}}{\mathcal{Z}_{e}}-\frac{\mathcal{Z}_{e}}{\mathcal{Z}_{m}}\right) \sinh \left(\gamma_{e} \ell_{e}\right)\right]
\end{aligned}
$$

The subscripts $m$ and $e$ refer to the magnetic and electric sections respectively, the subscript $p$ to period; the lower signs apply when

$$
\cosh \left(\gamma_{m} \ell_{m}\right) \cosh \left(\gamma_{e} \ell_{e}\right)+0.5\left(\frac{\mathcal{Z}_{m}}{\mathcal{Z}_{e}}+\frac{\mathcal{Z}_{e}}{\mathcal{Z}_{m}}\right) \sinh \left(\gamma_{m} \ell_{m}\right) \sinh \left(\gamma_{e} \ell_{e}\right)<-1
$$

Since $\gamma_{m}$ and $\gamma_{e}$ are both proportional to $p$ they are real or imaginary simultaneously. The other parameters in (2a) are all real, hence the expression within the absolute mark, $\|$, is always real. It can be less then -1 however. Because $\cosh (\psi) \geq-1$ for any $\psi$, real or imaginary, it is necessary to switch the signs of the diagonal elements explicitly whenever this occurs. The matrix for the 14 period string becomes:

$$
\left[h_{14 p}\right]=\left[\begin{array}{cc}
\cosh (14 \psi) & \pm \mathcal{Z}_{p} \sinh (14 \psi) \\
\pm \frac{\sinh (14 \psi)}{\mathcal{Z}_{p}} & \cosh (14 \psi)
\end{array}\right]
$$

and that for the whole kicker:

$$
[h]=\left[\begin{array}{cc} 
\pm \cosh (\Psi) & \mathcal{Z}_{p} \sinh (\Psi) \\
\frac{\sinh (\Psi)}{\mathcal{Z}_{p}} & \pm \cosh (\Psi)
\end{array}\right]
$$

where

$$
\begin{gathered}
\cosh (\Psi)=\left| \pm \cosh \left(\gamma_{m} \ell_{m}\right) \cosh (14 \psi) \pm 0.5\left(\frac{\mathcal{Z}_{m}}{\mathcal{Z}_{p}}+\frac{\mathcal{Z}_{p}}{\mathcal{Z}_{m}}\right) \sinh \left(\gamma_{m} \ell_{m}\right) \sinh (14 \psi)\right| \\
\mathcal{Z}_{k}=\frac{\mathcal{Z}_{m}}{\sinh (\Psi)}\left[ \pm \sinh \left(\gamma_{m} \ell_{m}\right) \cosh (14 \psi) \pm 0.5\left(\frac{\mathcal{Z}_{m}}{\mathcal{Z}_{p}}+\frac{\mathcal{Z}_{p}}{\mathcal{Z}_{m}}\right) \cosh \left(\gamma_{m} \ell_{m}\right) \sinh (14 \psi) \mp\right. \\
\left.\mp 0.5\left(\frac{\mathcal{Z}_{m}}{\mathcal{Z}_{p}}-\frac{\mathcal{Z}_{p}}{\mathcal{Z}_{m}}\right) \sinh (14 \psi)\right]
\end{gathered}
$$

and where the lower signs apply whenever

$$
\pm \cosh \left(\gamma_{m} \ell_{m}\right) \cosh (14 \psi) \pm 0.5\left(\frac{\mathcal{Z}_{m}}{\mathcal{Z}_{p}}+\frac{\mathcal{Z}_{p}}{\mathcal{Z}_{m}}\right) \sinh \left(\gamma_{m} \ell_{m}\right) \sinh (14 \psi)<-1
$$

The eigenvalues of the matrices in (2) and (3) are complex if $\cosh (\psi) \leq-1$, and real if $\cosh (\psi)>1$; those of the matrix in (4) are complex if $\cosh (\Psi) \leq 1$ and real if $\cosh (\Psi)>1$. The associated $\mathcal{Z}$ 's are real if the eigenvalues are complex and imaginary if they are real. 
The intervals of $p=j \omega$ for which the eigenvalues are imaginary are called passbands because wave propagation is possible in these intervals, those for which they are real are called stopbands.

It is not necessary to disregard the possibility of small parameter variations in the numerical approach because each section can be treated individually in the calculations. Multiplication of the matrices $\left[h_{k}\right]$ of the individual section yields the transfer matrix $[h]$ of the kicker:

$$
[h]=\prod_{k=1, k \max }\left[h_{k}\right]
$$

where the subscript $k$ is the section number. Once the elements of the $h$-matrix are known they can be used to calculate parameters of interest. E.G. for the voltage $U_{0}$, and the current $I_{o}$ at the input of the kicker in terms of source voltage, $U_{s}$ and the impedances of the source $Z_{s}$ and the termination $Z_{t}$ one finds:

$$
\left[\begin{array}{c}
U \\
I
\end{array}\right]_{0}=\left[\begin{array}{c}
Z_{\text {in }} /\left(Z_{\text {in }}+Z_{s}\right) \\
1 /\left(Z_{\text {in }}+Z_{s}\right)
\end{array}\right]\left[U_{s}\right]
$$

where

$$
Z_{\text {in }}=\frac{-h_{22} Z_{t}+h_{12}}{h_{21} Z_{t}-h_{11}}
$$

is the impedance measured at the input of the kicker if it is terminated in an impedance $Z_{t}$. One may also define a reflection coefficient $r f l$ which indicates what fraction of the incident source signal is reflected at the kicker's input and a transmission coefficient trm which indicates what fraction of the incident signal reaches the termination:

$$
\begin{aligned}
r f l & =\frac{Z_{\text {in }}-Z_{s}}{Z_{\text {in }}+Z_{s}} \\
t r m & =\frac{h_{11} Z_{\text {in }}+h_{12}}{h_{21} Z_{s}+h_{22}}
\end{aligned}
$$

Ideally $r f l=0$, this requires via $Z_{\text {in }}=Z_{s}$ :

$$
Z_{t}=\frac{h_{11} Z_{s}+h_{12}}{h_{21} Z_{s}+h_{22}}
$$




\section{Particle Deflection}

The change in direction of motion of a particle that passed through the kicker is the sum of the deflections it received in its individual elements. In calculating these deflection we assume that the deflecting fields are uniform and that the gain or loss of energy due to the longitudinal component of the electric field $\left(<10^{-4}\right)$ is sufficiently small to be disregarded. The deflection in a section is then proportional to its integrated deflecting field. The electric component of that field is proportional to the local voltage $U(z)$, its magnetic component is proportional to the local current $I(z)$, the relations between field and voltage and current are invariant over the length of the section under consideration. Solution of the telegraph equations yields

$$
\left[\begin{array}{c}
u(z, t) \\
i(z, t)
\end{array}\right]=\exp (j \omega t)\left[\begin{array}{cc}
\cos (\gamma z) & -j \mathcal{Z} \sin (\gamma z) \\
-j \frac{\sin (\gamma z)}{\mathcal{Z}} & \cos (\gamma z)
\end{array}\right]\left[\begin{array}{c}
U \\
I
\end{array}\right]
$$

where $U$ and $I$ are the (complex) amplitudes of voltage and current at the entrance boundary of the section. The effect of the field on a particle depends in the direction of the particle's velocity $v$, we count $v$ positive if the particle moves in the direction from source to termination, negative if it moves from termination to source. A particle of velocity $v>0$ that passes $z=0$ at time $t_{0}$ reaches $z$ at time $t=t_{o}+z / v$, substitution of this $t$ and integration over the section length $I$ yields

$$
\left[\begin{array}{c}
\int U\left(z, t_{o}\right) d x \\
\int I\left(z, t_{o}\right) d z
\end{array}\right]=\frac{\exp \left(j \omega t_{o}\right)}{1-\left(\frac{\omega}{\gamma v}\right)^{z}}\left[\begin{array}{cc}
c i & -j \mathcal{Z} s i \\
-j s i / \mathcal{Z} & c i
\end{array}\right]\left[\begin{array}{c}
U \\
I
\end{array}\right]
$$

where

$$
\begin{aligned}
& c i=\exp (j \omega I / v) \sin (\gamma I)-j \frac{\omega}{\gamma v}\{1-\exp (j \omega I / v) \cos (\gamma I)\} \\
& s i=1-\exp (j \omega 1 / v) \cos (I \gamma)+j \frac{\omega}{\gamma v} \exp (j \omega I / v) \sin (I \gamma)
\end{aligned}
$$

The expressions must be modified to read

$$
\left[\begin{array}{l}
\int U\left(z, t_{o}\right) d z \\
\int I\left(z, t_{o}\right) d z
\end{array}\right]=\frac{\exp \left\{j \omega\left(t_{o}-I / v\right)\right\}}{1-\left(\frac{\omega}{\gamma v}\right)^{2}}\left[\begin{array}{cc}
c i & -j \mathcal{Z} s i \\
-j s i / \mathcal{Z} & c i
\end{array}\right]\left[\begin{array}{l}
U \\
I
\end{array}\right]
$$

for particle with $v<0$; such particles enter at $z=I$ at time $t_{o}$ and reach $z$ at time $t=t_{o}-I / v+z / v, 0 \leq z \leq I$. Inspection of Fig. 2 shows that the electric component 
of the deflecting field is given by $E=U / w$, while its magnetic component follows from $B=\mathcal{L} I / w$, where $w$ is the width of the aperture. The integrated deflecting field in the $k^{\text {th }}$ section is therefore

$$
\left[\begin{array}{l}
\int E\left(z, t_{k}\right) d z \\
\int B\left(z, t_{k}\right) d z
\end{array}\right]=\left[\frac{1}{w} \frac{\exp \left(j \omega t_{k}\right)}{1-\left(\frac{\omega}{\gamma v}\right)^{2}}\left[\begin{array}{cc}
c i & -j \mathcal{Z} s i \\
-j(\mathcal{L} C)^{1 / 2} & \text { si }
\end{array}\right]\right]_{k} \prod_{i=k-1,1}\left[h_{i}\right]\left[\begin{array}{c}
U \\
I
\end{array}\right]_{0}
$$

where $t_{k}$ labels the time at which the particle enters the $k^{\text {th }}$ section. Evidently for $v>0$ :

$$
\begin{aligned}
& t_{1}=t_{o} \\
& t_{2}=t_{1}+I_{1} / v \\
& t_{k}=t_{k-1}+I_{k-1} / v
\end{aligned}
$$

where $t_{o}$ is the time at which the particle enters the kicker (at its source end). For $v<0$ :

$$
\begin{aligned}
t_{k m x} & =t_{o}-I_{k m x} / v \\
t_{k m x-1} & =t_{k m x}-I_{k m x-1} / v \\
t_{k} & =t_{k+1}-I_{l} / v
\end{aligned}
$$

where $t_{o}$ is still the time at which the particle enters the kicker but now at its termination end.

The deflecting field produces a deflection

$$
\Delta \varphi=\left(\int E d z-v \int B d z\right) /(B \rho)
$$

for particles with magnetic rigidity $B \rho$; the total deflection due to the kicker may therefore be described by

$$
\Delta \phi\left(t_{o}\right)=(B \rho)^{-1} \sum_{k=1, k m x}\left\{\left[\int E\left(z, t_{k}\right) / d x\right]_{k}-v\left[\int B\left(z, t_{k}\right) d z\right]_{k}\right\}
$$




\section{Some Results}

For the first exercise we consider a kicker straight from the drawing board. We estimate, using the dimensions of fig. 2 and $\mu_{r}>100, \epsilon_{r} \cong 12$ in the ferrite and $\epsilon_{r}=100$ in the high permittivity ceramic:

Table II: Section Characteristics of Nominal Kicker

$\begin{array}{lll} & \text { Inductive Section } & \text { Capacitive Section } \\ \ell & 5 & 2.5[\mathrm{~cm}] \\ \mathcal{L} & 1.225 & 0.2282[\mu \mathrm{H} / \mathrm{m}] \\ \mathcal{C} & 0.3785 & 3.972[\mathrm{nF} / \mathrm{m}] \\ \mathcal{Z}=(\mathcal{L} / \mathcal{C})^{1 / 2} & 56.89 & 7.580[\Omega] \\ v=(\mathcal{L C})^{-1 / 2} & 4.644 \times 10^{7} & 3.322 \times 10^{7}[\mathrm{~m} / \mathrm{sec}] \\ \text { number } & 15 & \text { sections } \\ \text { number } & & 14 \text { sections }\end{array}$

The results are given in the form of graphs and bundled in three groups. The number of possible graphs is very large, therefore only the ones that describe the critical features best are provided here, any others can always be made on request. All 2048 points in each of these graphs are individual point symbols. The first group describes the nominal kicker, its sections are described in Table II. It consists of 14 periods with an added half length inductive section at each end. These added half sections account for the fact that all 15 inductive sections have the same physical length. Figs. 3 and 4 show the behavior of the real and imaginary components of the kicker's characteristic impedance $\mathcal{Z}_{c h}$ as function of frequency, Fig. 4 was added to show that behavior in more detail near the lower edge of the first major stopband at $122.75 \mathrm{MHz}$. The maximum $\mathcal{Z}_{c h}$ shown was limited to $100 \mathrm{Ohm}$ in order to have a reasonable resolution, some values were much larger than that. It may be seen that either $\operatorname{Imag}\left(\mathcal{Z}_{c h}\right)=0$ or $\operatorname{Real}\left(\mathcal{Z}_{c h}\right)=0$, as was expected from the analytical approach. The 14 mini stopbands between $12.012 \mathrm{MHz}$ and $122.75 \mathrm{MHz}$ are stem from the mismatch generated by the added half length inductive sections. This is evident from a comparison with Fig. 10 which shows the behavior of $\mathcal{Z}_{c h}$ for a kicker with the same 14 period structure, but without the added half sections (and which therefore begins and 
ends with half length inductive sections). Figs. 5-9 represent results in the time domain for the nominal kicker. The source voltage is a square wave which is transformed to the frequency domain using $f f t$ with $n$ points and a maximum frequency freqm. It appears that the choice of that maximum frequency affects the particle deflection considerably if it is chosen beyond the lower edge of the first major stopband, and hardly if it stays below that edge. While the deflection curve shows the expected reasonably smooth behavior if the maximum frequency is below that edge, its successive points, which are half a period of the maximum frequency apart in time, are shifted by large and equal amounts in opposite directions away from the average if it is above it. Numerical experimentation shows that the effect becomes worse with increasing maximum frequency and that it becomes increasingly concentrated near the instants of change of state if $n$, the number of harmonics, is increased. We have time nor space to investigate this matter more fully and suppress the effect by keeping the maximum frequency just below the band edge, i.e., at $120 \mathrm{MHz}$. Fig. 5 shows the (normalized) source voltage, the deflection factor, and the transmission and reflection coefficients during a full period, Fig. 6 shows the same parameters with a much reduced time scale in the vicinity of the step in the square wave of the source voltage. Deflection, transmission and reflection vary somewhat immediately after the step but settle down to practically constant values after a $0.5 \mu \mathrm{sec}$ or so. Taking the deflection at the midpoint of the step as reference we calculate the relative deflection error as function of time after the instant that the source voltage reaches full value; as may be seen from Fig. 7 the deflection is within \pm 0.01 of nominal value some $80 \mathrm{nsec}$ after the source voltage has reached its nominal value. The scatter in the datapoints will lead to emittance blowup due to differential deflection of successive slices of the bunch. The consequences of choosing the maximum frequency beyond the lower band edge are shown in Figs. 8 and 9.

The scatter in the points of the transmission and reflection curves suggests that the basic structure can be improved. Removing the 'extra' half lengths of the first and last sections of the nominal kicker one obtains a strictly periodic structure. The characteristic impedance of the new structure is lower than that of the original one, thus we decrease the capacitance of the capacitive sections from $\mathcal{C}=3.972 \mathrm{nF} / \mathrm{m}$ to $\mathcal{C}=3.528 \mathrm{nF} / \mathrm{m}$, to make $\mathcal{Z}_{c h}=25 \mathrm{Ohm}$ at very low frequencies. The characteristics of this new structure are described by Figs. 10-13. The behavior of the characteristic impedance as function of 
frequency is given by Fig. 10. It is clear that the mini stopbands have disappeared and that the lower edge of the first stopband is moved up to about $130 \mathrm{MHz}$. Increasing the lengths of the individual sections from $5 \mathrm{~cm}$ and $2.5 \mathrm{~cm}$ to $5.365 \mathrm{~cm}$ and $2.683 \mathrm{~cm}$ in order to keep the overall length at $1.1 \mathrm{~m}$ would return the lower edge frequency to about 120 $\mathrm{MHz} . \mathcal{Z}_{c h}$ is real is the passband and imaginary in the stopband, as expected. Figs. 11-13 are directly comparable with Figs. 5-7 for the nominal kicker. One notes that there is still some scatter in the points of the deflection, transmission and reflection curves. The nominal deflection is reduced to 0.940 of the previous case, because the new structure is shorter: the total length of the magnetic sections was $75 \mathrm{~cm}$. The principal gain is that the deflection is within \pm 0.01 of the nominal one within 50 nsec after the source voltage reaches its nominal value and that the emittance blow up is less and occurs over shorter time-interval.

The results for the continuous kicker are still better. Its $\mathcal{Z}_{c h}$ is independent of frequency so we did not graph it. The behavior of its deflection and transmission in time is described by Figs. 14-16, its reflection coefficient, which is $r f l \cong 0 \pm 10^{-18}$ for all points, is not shown. Its deflection is a factor 1.320 larger than tat of the nominal case because its deflecting field is available over its full length rather than predominantly in the magnetic sections. The value of freqm may be chosen arbitrarily since there are no stopbands, $120 \mathrm{Mhz}$ was chosen for this particular case to provide for easy comparison with the results for the segmented kickers. Fig. 15 shows an unexpected variation of the transmission coefficient in the vicinity of the switching points. Since I have not been able to trace the origin I suspect the limited numerical precision of the method. Fig. 16 shows similar unexpected behavior of the relative deflection error and I blame the same cause, at least for now, but the error is within \pm 0.005 within $50 \mathrm{nsec}$ after the source voltage has reached its nominal value even so. Figs. 17-18 show that mismatches at the source and/or load ends of the kicker tend to spoil the performance: the earliest instant at which the deflection error becomes acceptable is shifted to later times. 


\section{Discussion}

The results obtained here may well differ from those obtained by measurement from the physical kicker because of lack of correspondence between model and reality.

The first obvious deviation is that the cross-sectional dimensions of the real kicker elements are not small compared to their lengths so that the coupling between successive elements is more complicated than is assumed in the model. However, these dimensions are not large compared to the lengths either: the length of a period (the sum of the lengths of an inductive and an capacitive element) is $7.5 \mathrm{~cm}$, about 1.5 times the larger cross-sectional dimensions. The passband/stopband structure found in the calculations may therefore still show up in reality.

A second objection is that the dispersion due to the non-uniform filling of the space between outer and inner conductor(s) mentioned in the introduction has been disregarded. The very simple model used to demonstrate the existence of this effect showed that it becomes increasingly noticeable for frequencies above $1 \mathrm{Mhz}$; it is difficult to estimate how serious it might be in the physical kicker. This would require a solution of Maxwell in three dimensional space + time; considerable work has been done, but is not finished yet.

A third deviation is that the ferrite and the dielectric ceramic are supposed to be free of dispersion and losses in the model, while in fact such dispersion and losses are present. Both effects can be incorporated in the model, the dispersion fairly easily, the losses only by further extension of the computer codes ( $\gamma$ and $\mathcal{Z}$, and consequently all matrix elements, change from purely real or purely imaginary quantities into complex ones, the determinant products of the matrices do no longer equal 1). Rough estimates suggest that both effects are relatively small within the frequency range considered here. They will however reduce the very large swings in the so-called mini-stopbands of the first kicker.

The connectors at the ends of the kicker, which connect it to the cables coming from the power source and to the load resistor were not considered, although they may add, either intentionally or unintentionally, inductance and/or capacitance to the first and last kicker elements, changing the quality of the matches. E.g., if the connectors add sufficient capacitance they may convert the first and last inductive half elements of the nominal 
kicker into half periods, converting it to a more nearly periodic structure but one with 15 periods instead of 14 .

The oscillations in the time curves when frequencies beyond the lowest stopband edge are present can perhaps be explained as follows: for those frequencies successive periods in the structure oscillate in anti-phase with amplitudes that increase exponentially with the sequence number of the period. The particles pass through the whole structure in about $3 \mathrm{nsec}$, i.e., in a fraction of an oscillation period, and each particle sums the forces experienced in the successive structure periods. This sum is generally far from zero, even though the forces are in approximate anti-phase, because of the exponential increase in the field amplitudes. It switches sign every half period of oscillation thus particles which pass through a structure period $4 \mathrm{nsec}$ apart feel forces of opposite sign. The effect depends upon the frequency because the exponential growth depends on frequency. Generally there are several to many harmonics; one expects therefore at least partial cancellation to occur after some decoherence time. That decoherence time should decrease with increasing number of harmonics, concentrating the effect to the vicinity of the change-of-state points, precisely the time intervals of interest. One concern has been mentioned earlier: degradation of emittance because of differential deflection of successive bunch slices. This can be prevented by shaping of the waveform of the power pulse such that there are no objectionable frequencies, but doing so may well increase the net filling time. A second concern is the interaction between kicker and circulating bunched beam. There is a dipole coupling moment between the beam and the kicker structure because of the physical asymmetry in the cross-section of the beam-kicker configuration. The beam can therefore drive the individual structural periods of the kicker, and it is certain to do so because it contains components with frequencies within the stopbands. 


\section{Acknowledgment}

I wish to express my thanks to Brookhaven National Laboratory, the RHIC administration and in particular Dr. S. Ozaki for granting me the opportunity and means to work on this interesting problem. I also thank colleagues for discussions and their support. 


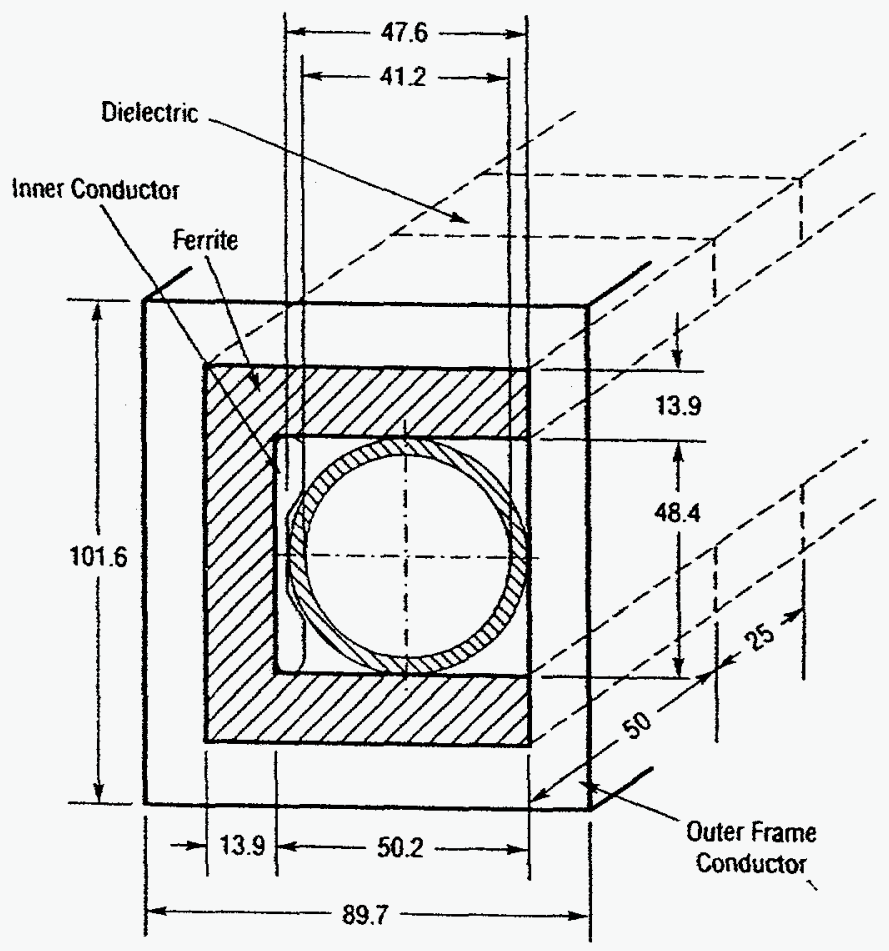

Fig. 1. Injection Kicker, alternating ferrite and dielectric sections. [mm]

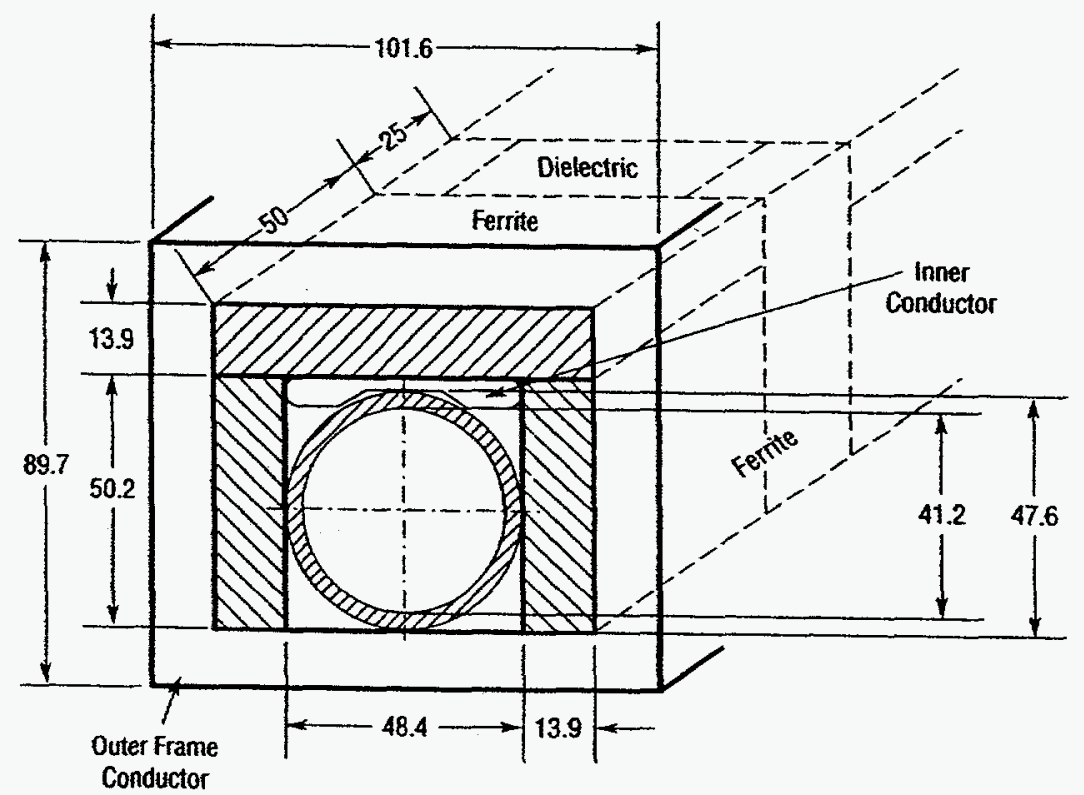

Fig. 2. RHIC Injection Kicker, continuous ferrite poleplates, alternating ferrite and dielectric backlegs. [mm] 
$1 M+14 *(E+M), \quad n=2048, \quad$ freqm $=1.5 e 8$

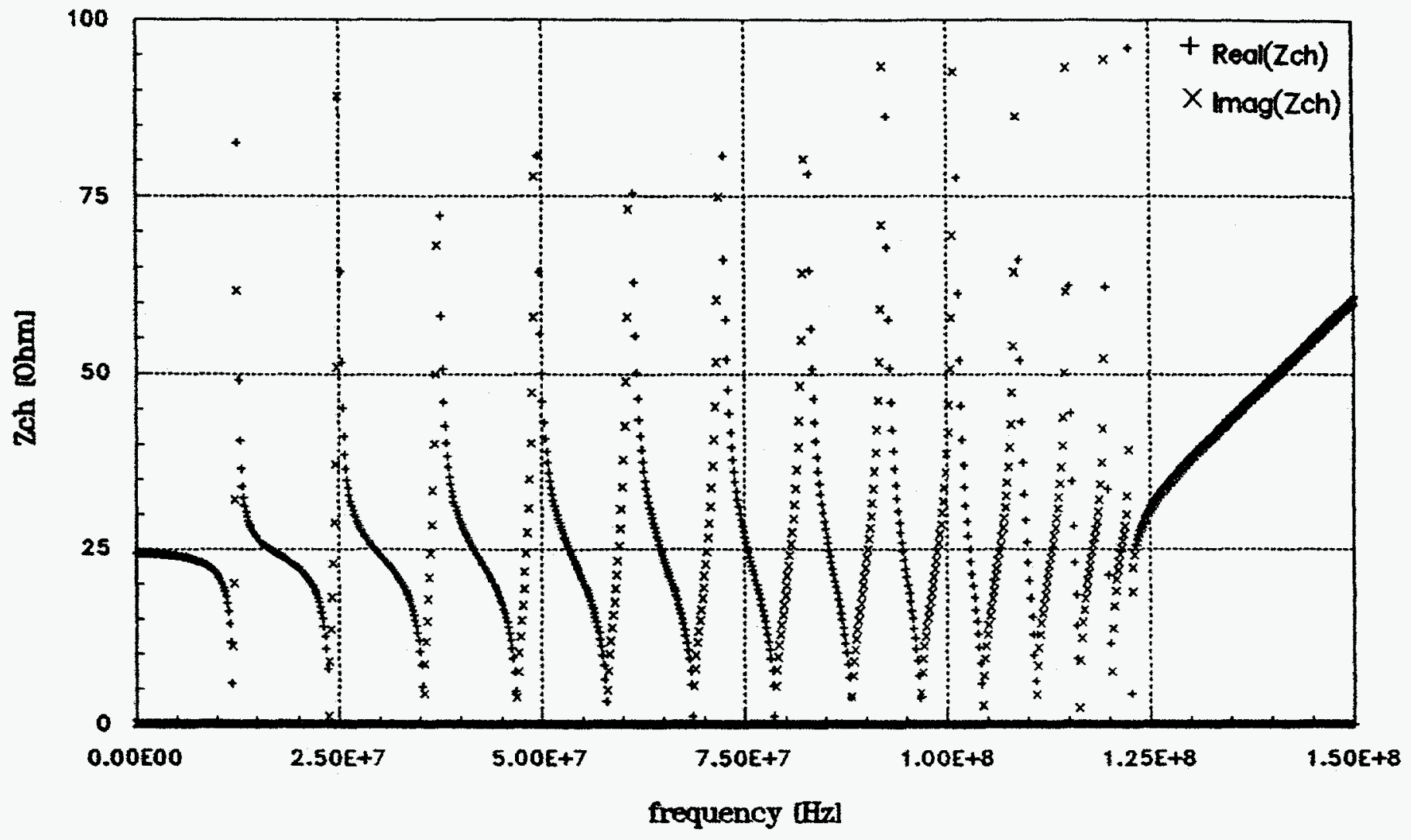

Fig. 3. Characteristic Impedance of nominal injection kicker as function of frequency, $0 \leq \nu \leq 150 \mathrm{MHz}$ 


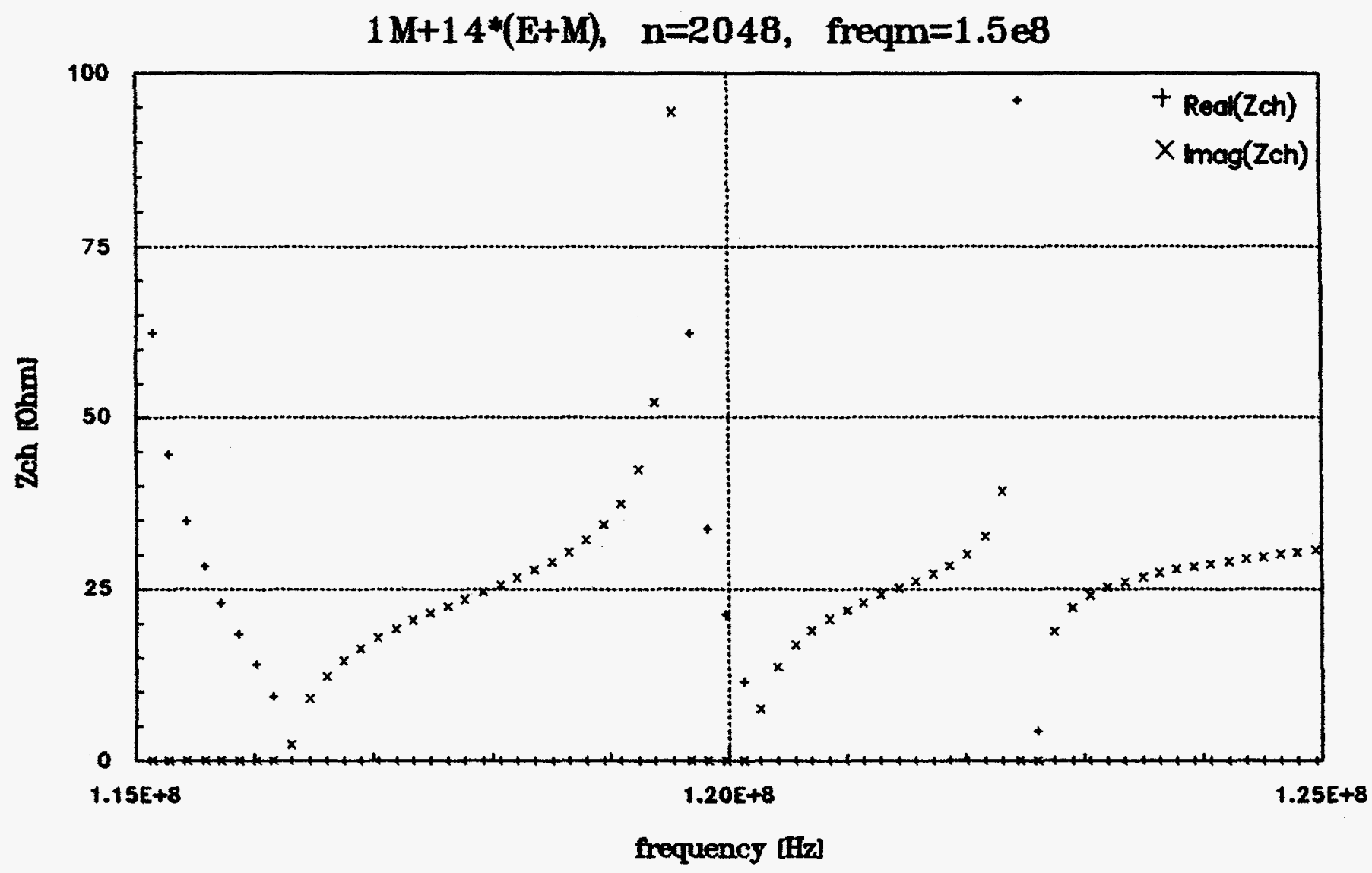

Fig. 4. Characteristic Impedance of nominal injection kicker as function of frequency, $115 \leq u \leq 125 \mathrm{MHz}$.

Note lower edge of major stopband at $\nu \cong 122.5 \mathrm{MHz}$. 
$1 M+14(E+M)$ sections, $n=2048$, freqm $=1.20 \mathrm{e} 8$

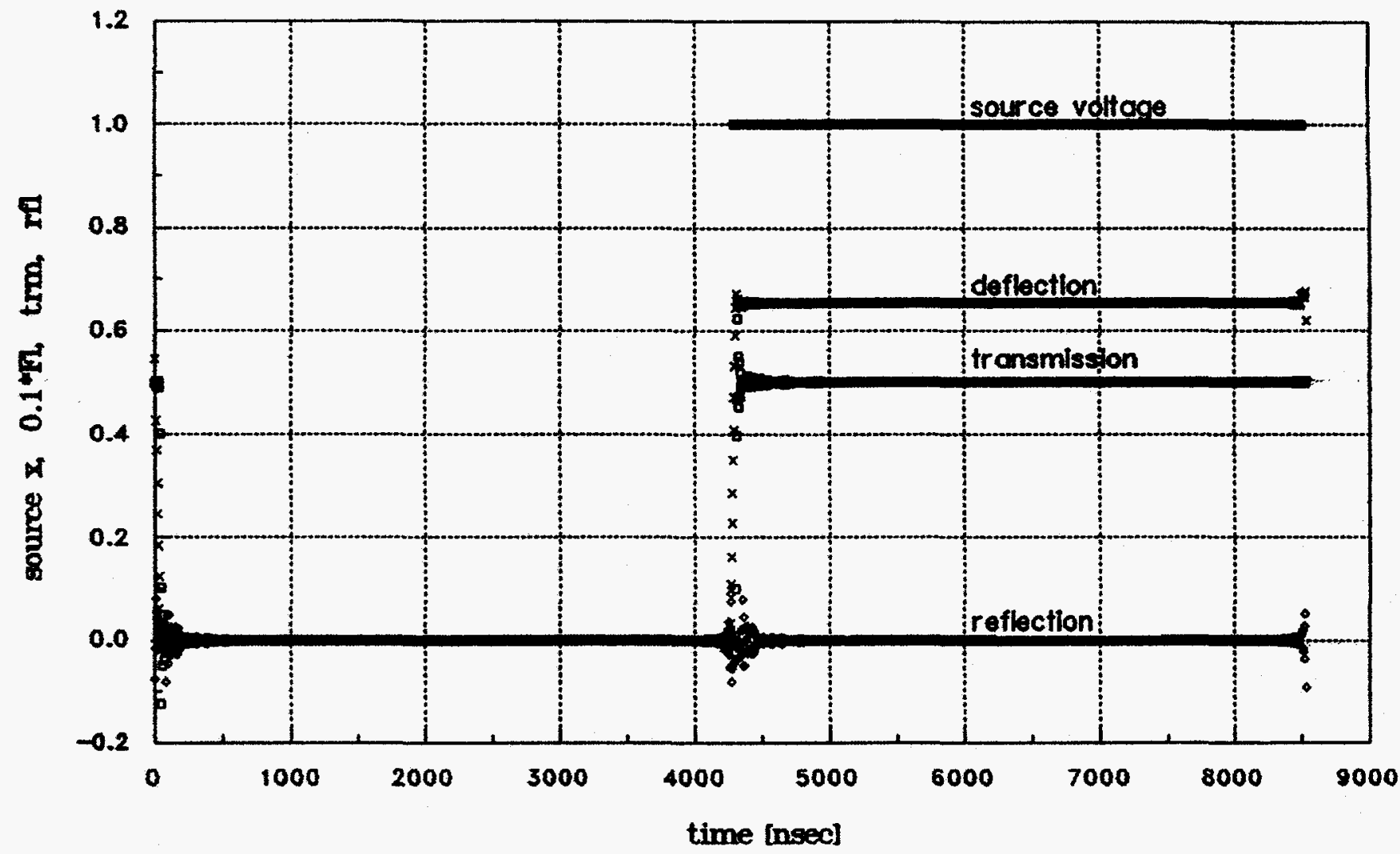

Fig. 5. Source voltage, deflection factor Fl, transmission factor trm and reflection factor rfl as functions of time for nominal kicker.

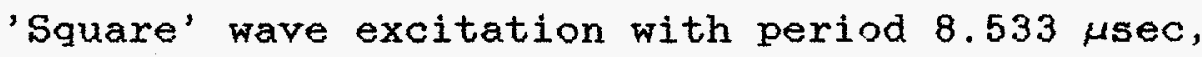
$v_{\text {max }}=120 \mathrm{MHz}$. 


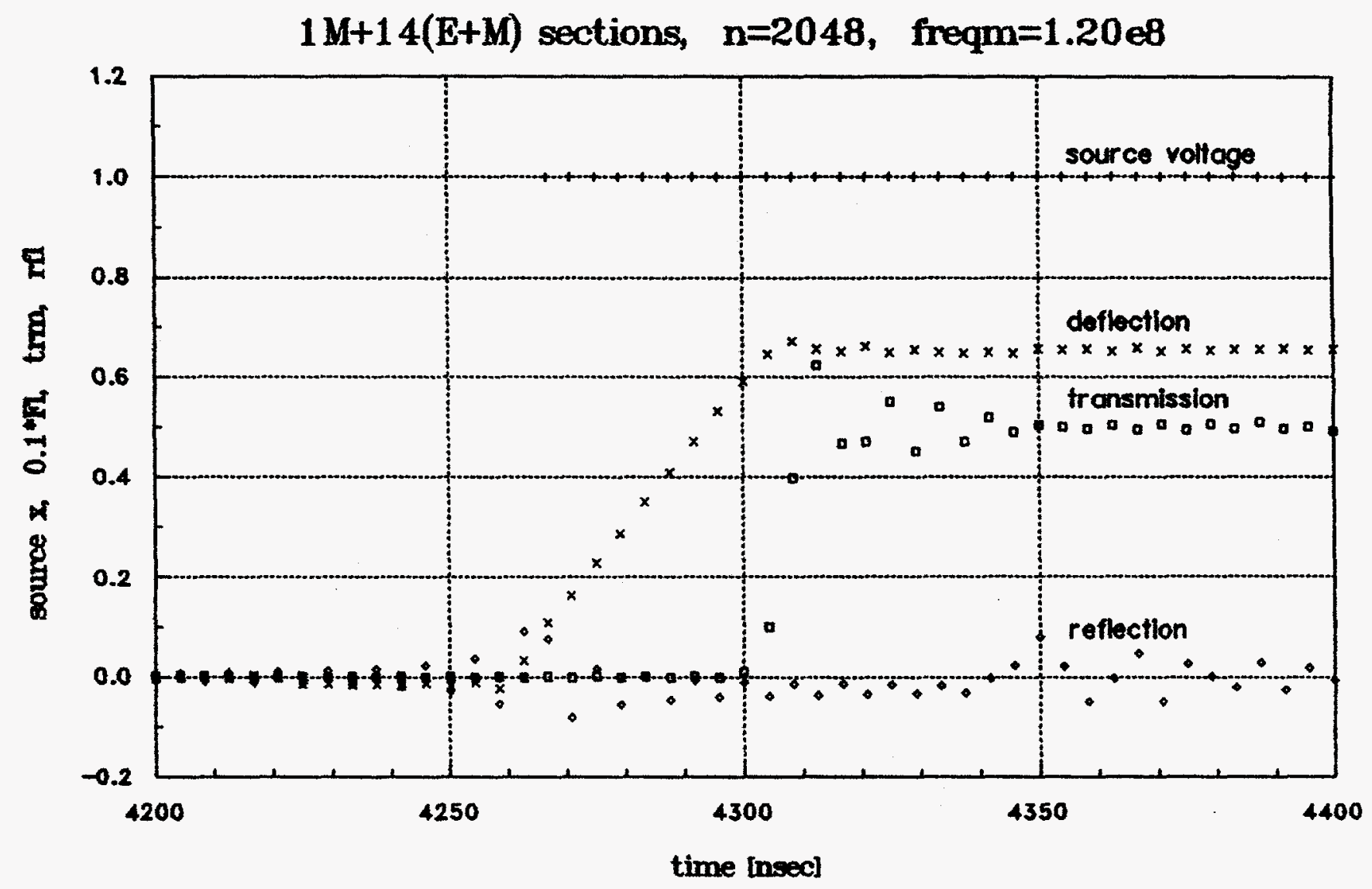

Fig. 6. Source voltage, deflection factor Fl, transmission factor trm and reflection factor rfl as functions of time for nominal kicker.

'Square' wave excitation with period $8.533 \mathrm{\mu sec}$, $\nu_{\max }=120 \mathrm{MHz}$. Time interval shown $4.2 \mu$ sec $\leq t \leq 4.4 \mu$ sec. Time interval between successive points of each curve: 4.17 nsec Note irregularities in $F 1$, trm and $\mathrm{rfl}$. 


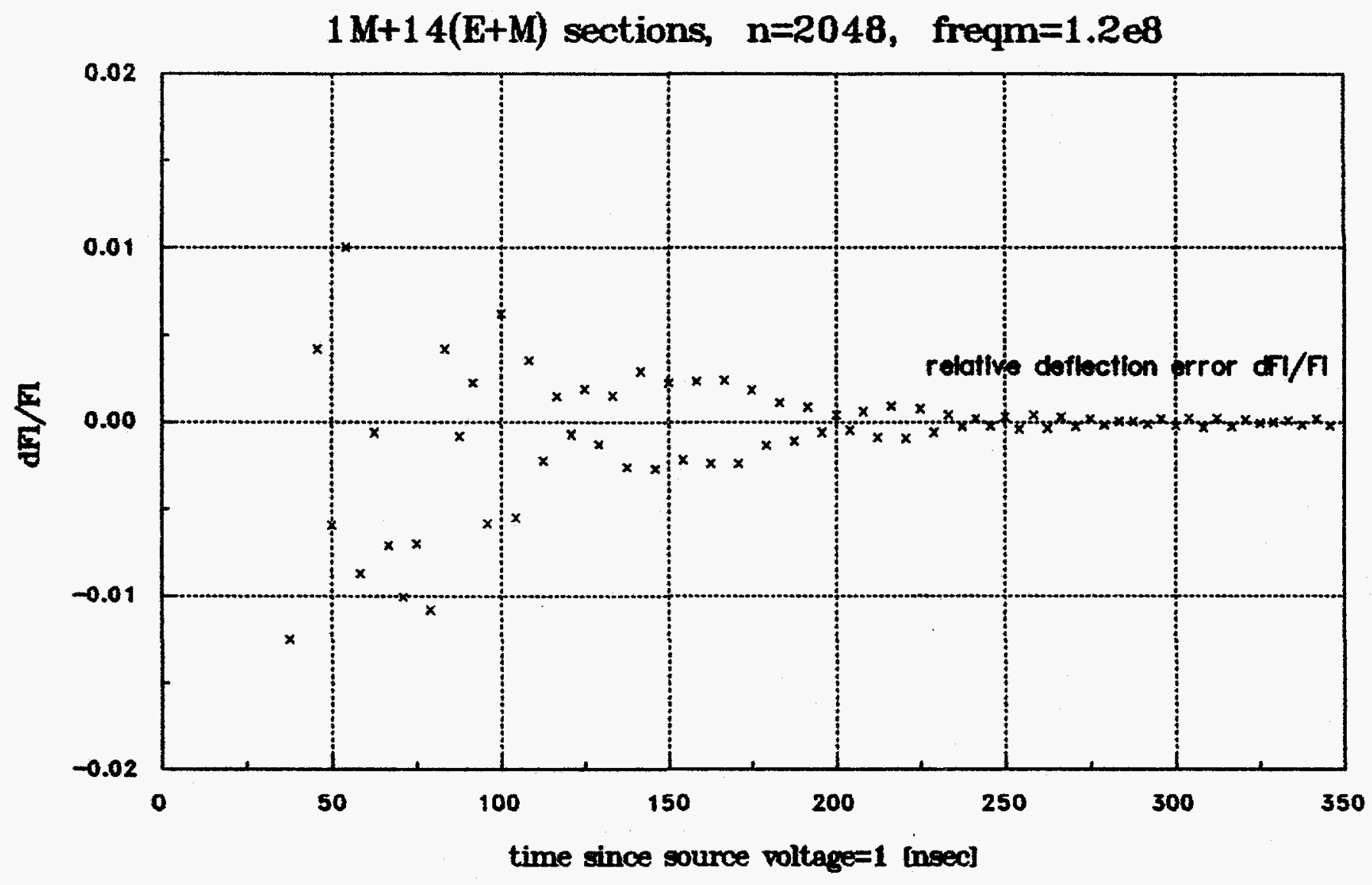

Fig. 7. Relative deflection error $\triangle F 1 / F 1$ during 350 nsec after the source voltage has reached nominal value. $v_{\max }=120 \mathrm{MHz}$

Time interval between successive points of curve: $\tau=4.17$ nsec. 


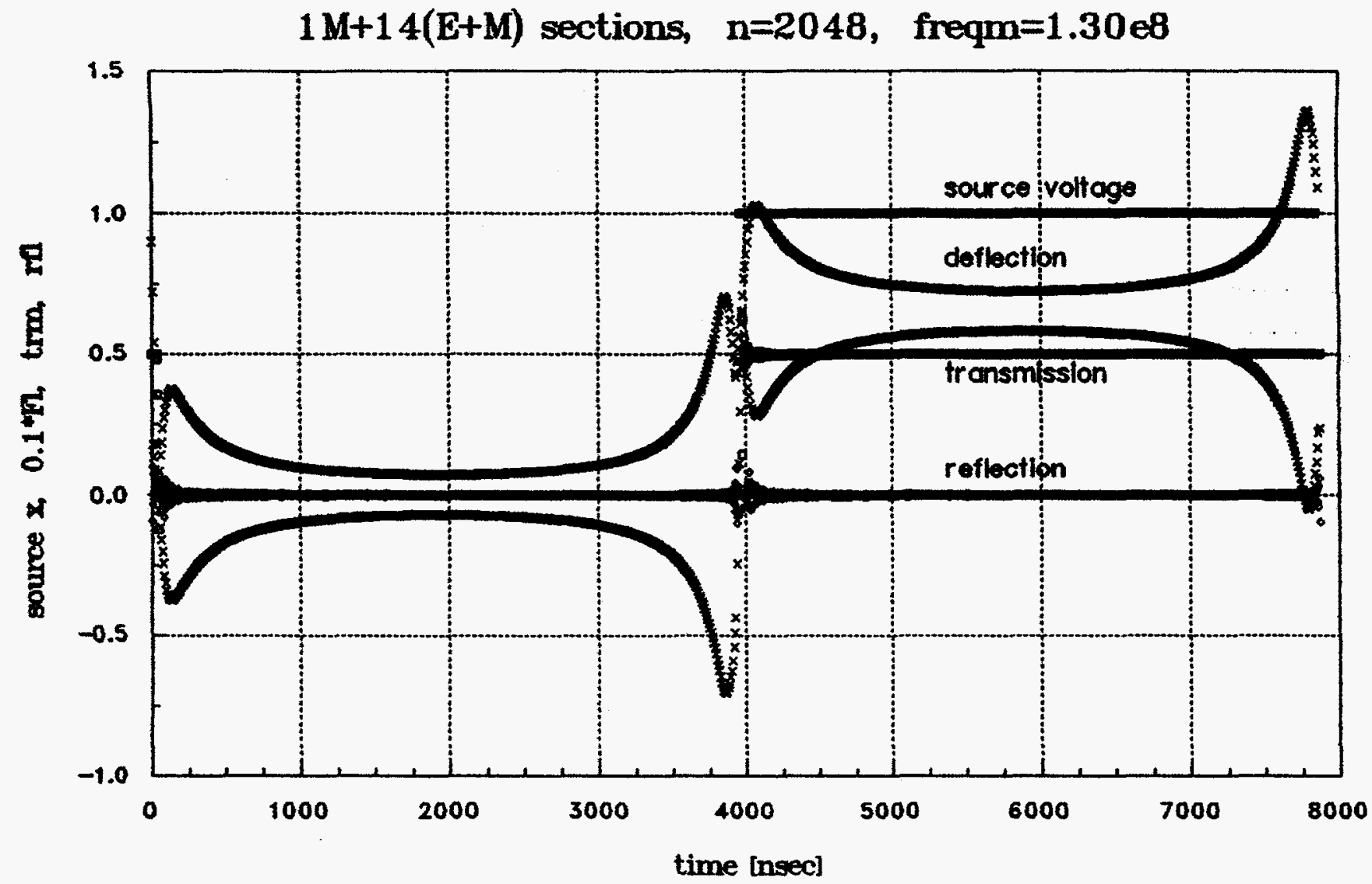

Fig. 8. Source voltage, deflection factor $F 1$, transmission factor trm and reflection factor rfl as functions of time for nominal kicker.

'Square' wave excitation with period 7.877 usec, $\nu_{\max }=130 \mathrm{MHz}$.

Note oscillations in deflection curve. 


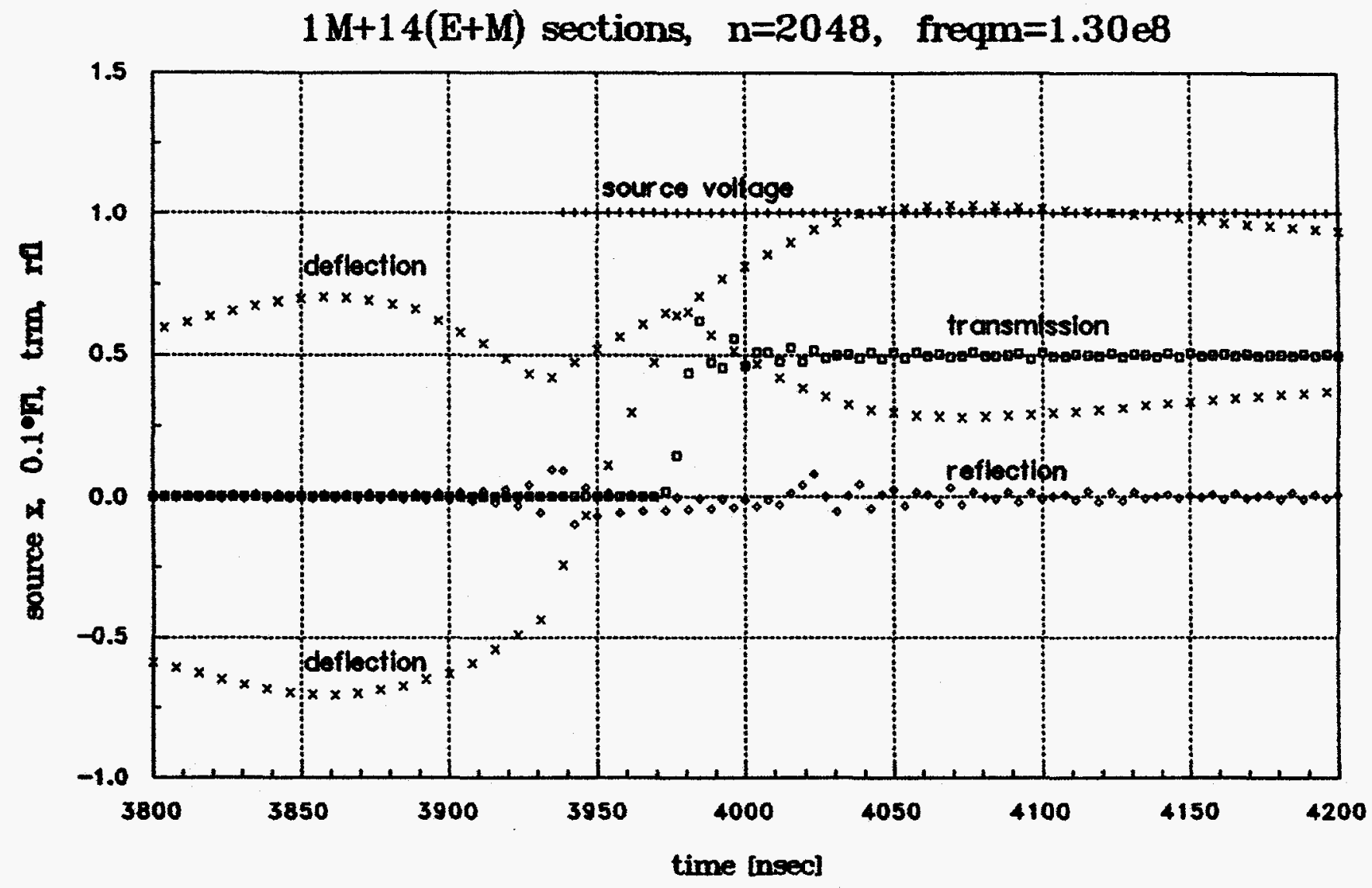

Fig. 9. Source voltage, deflection factor Fl, transmission factor trm and reflection factor rfl as functions of time for nominal kicker.

'Square' wave excitation with period $7.877 \mathrm{\mu sec}$, $v_{\max }=130 \mathrm{MHz}$. Time interval shown $3.8 \mu \mathrm{sec} \leq \mathrm{t} \leq 4.2 \mathrm{\mu sec}$. Time interval between successive points: $\tau=3.85 \mathrm{nsec}$. Note oscillations in Fl, trm and rfl. 


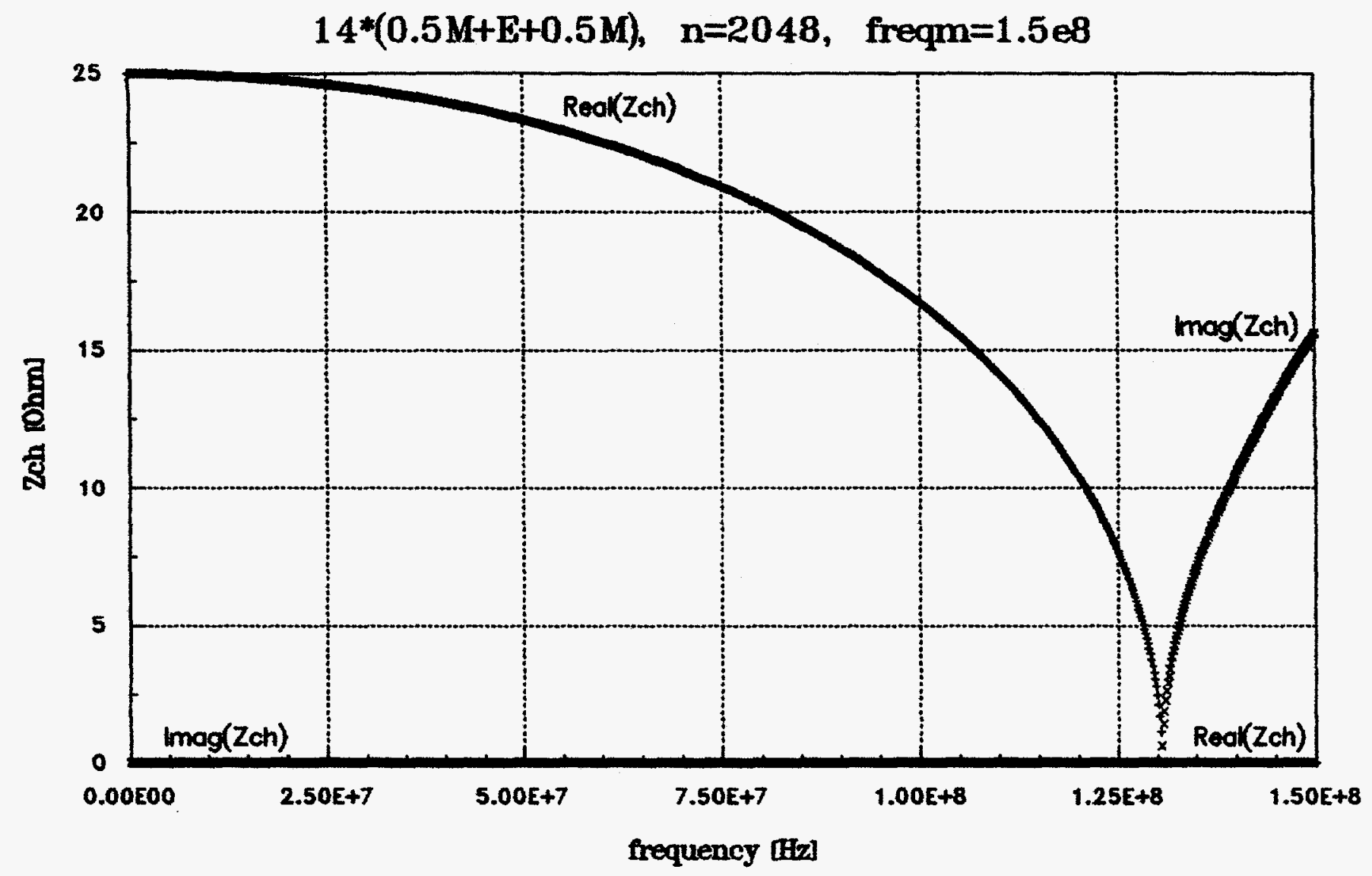

Fig. 10. Characteristic Impedance of periodic kicker with $Z_{c h}=25$ ohm as function of frequency, $0 \leq 2 \leq 150 \mathrm{MHz}$. Note absence of 'mini stopbands'. 


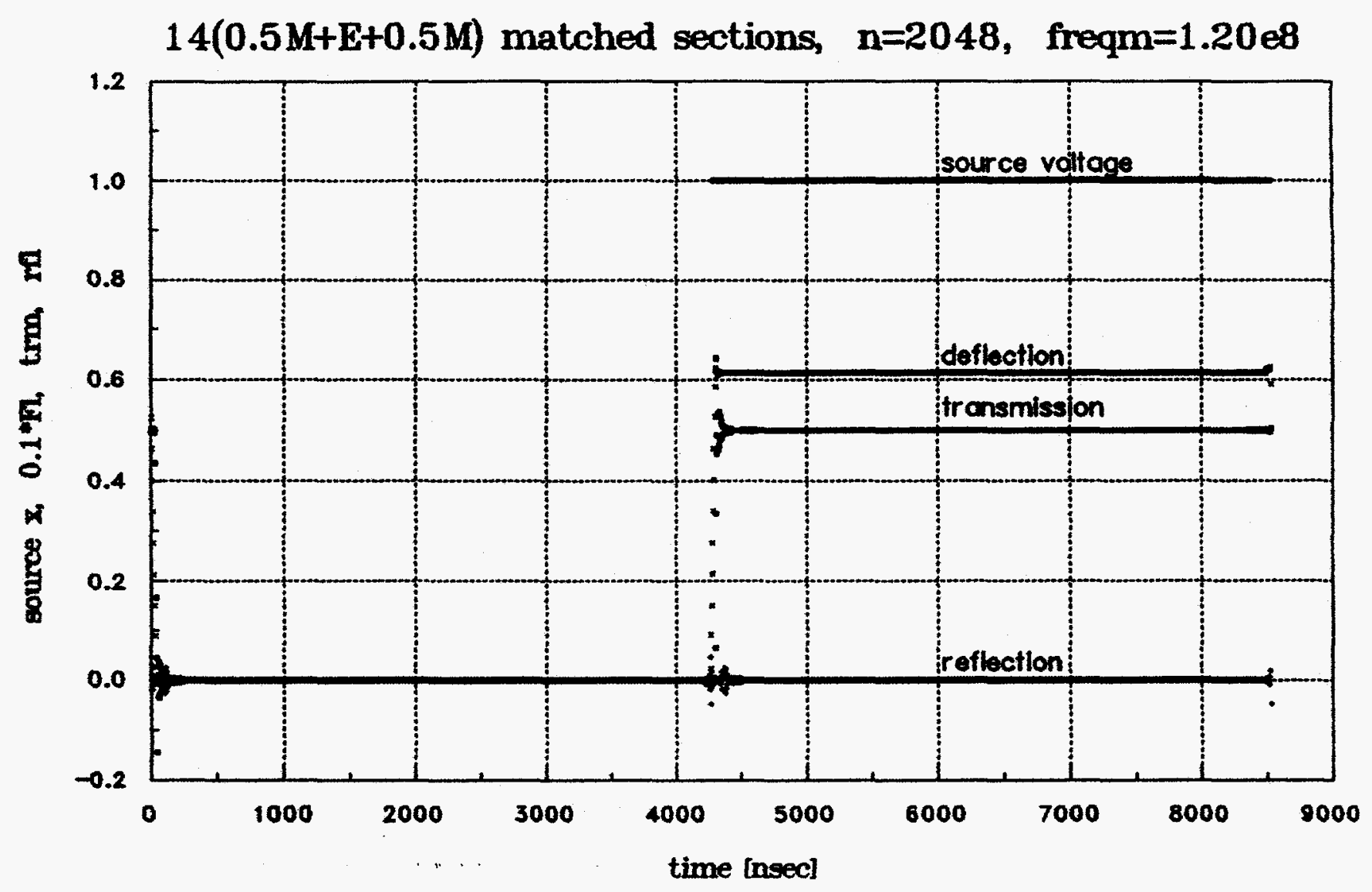

Fig. 11. Source voltage, deflection factor Fl, transmission factor trm and reflection factor rfl as functions of time for periodic and matched kicker.

'Square' wave excitation with period $8.533 \mathrm{\mu sec}$, $\nu_{\max }=120 \mathrm{MHz}$. 


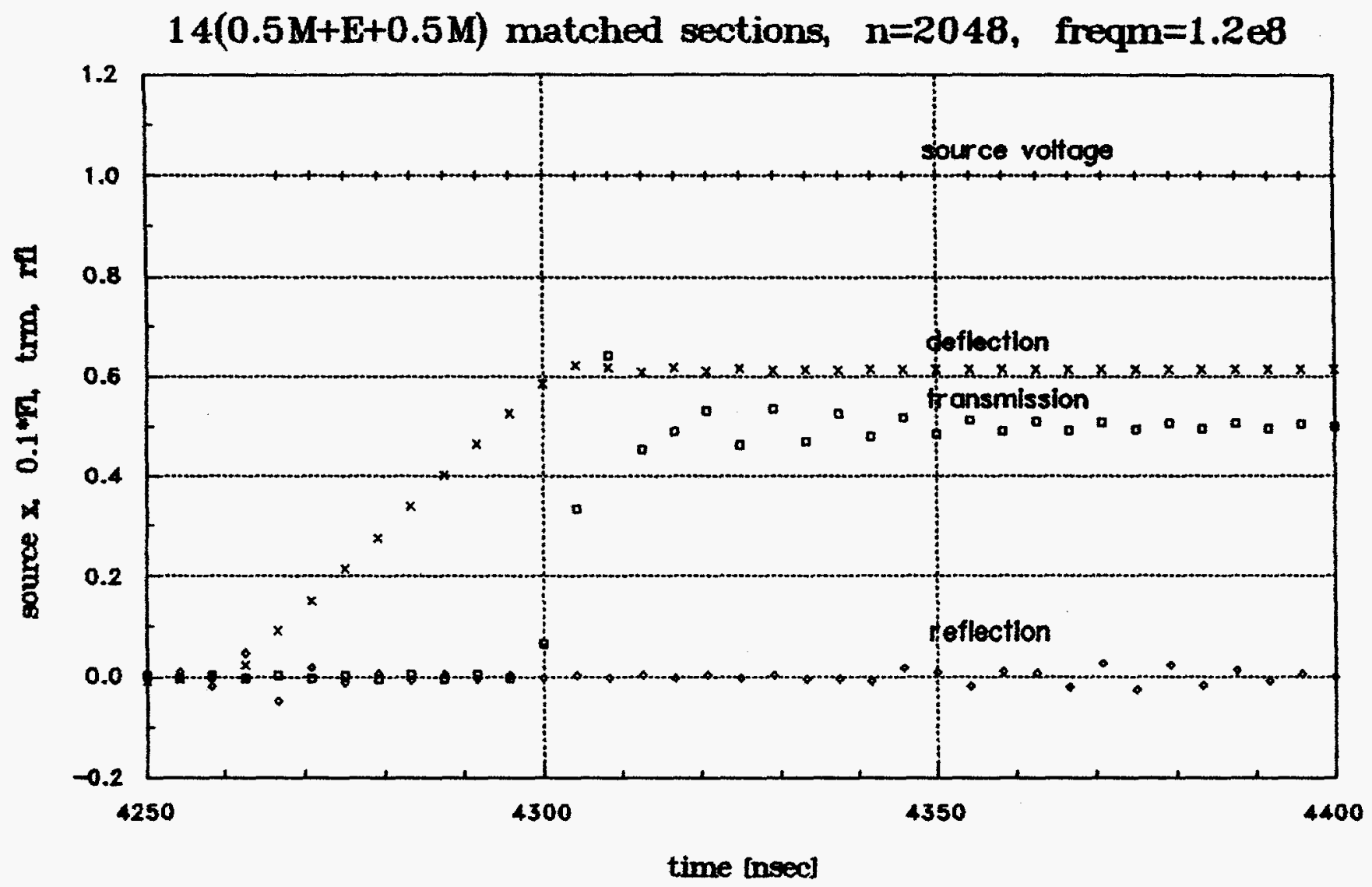

Fig. 12. Source voltage, deflection factor Fl, transmission factor trm and reflection factor $\mathrm{rfl}$ as functions of time for periodic and matched kicker.

'Square' wave excitation with period $8.533 \mu \mathrm{sec}$, $\nu_{\text {max }}=120 \mathrm{MHz}$. Time interval shown $4.25 \mathrm{\mu sec} \leq \mathrm{t} \leq 4.4 \mathrm{\mu sec}$.

Time interval between successive points of each curve: 4.17 nsec Note irregularities in $\mathrm{Fl}$, trm and $\mathrm{rfl}$. 


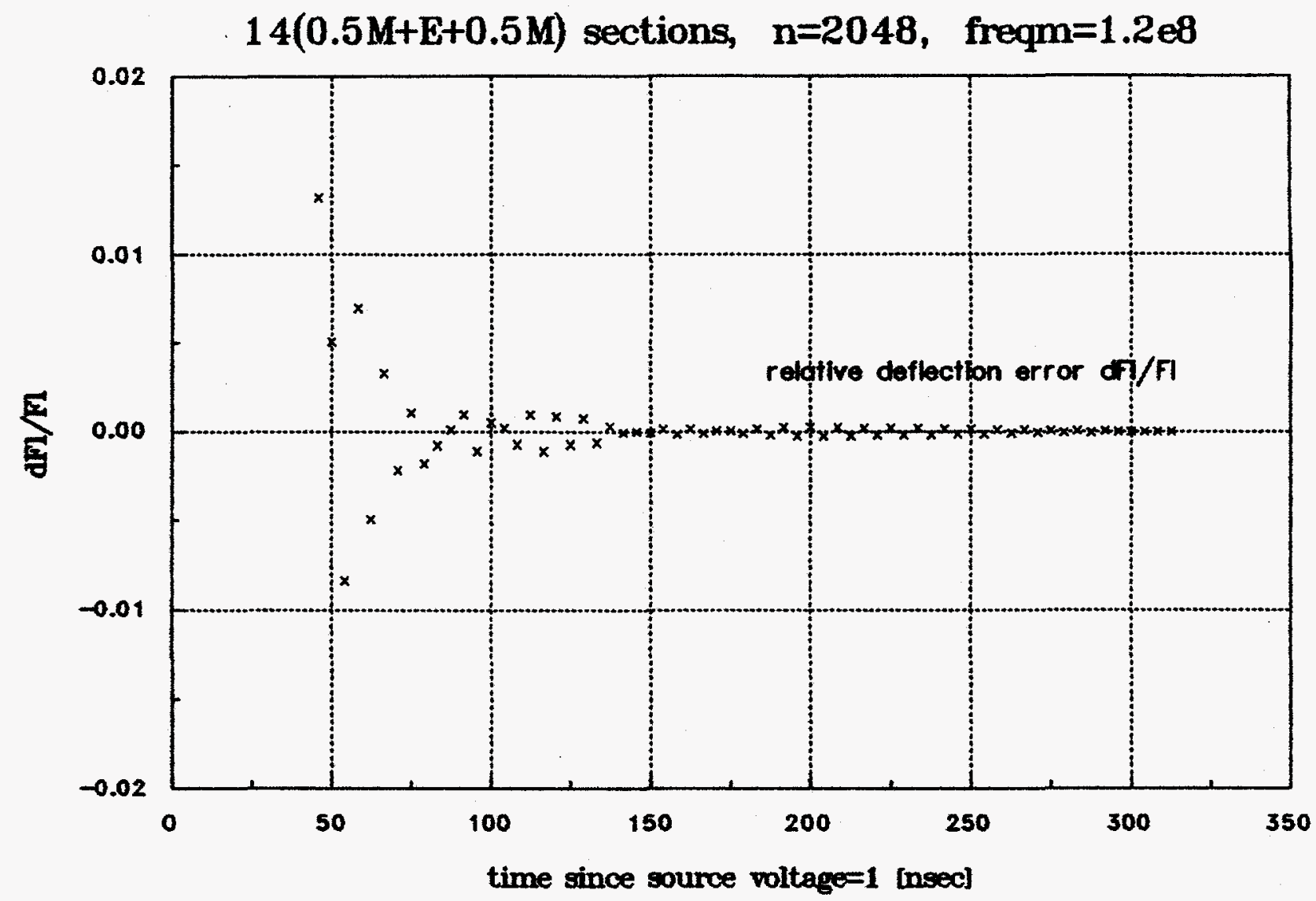

Fig. 13. Relative deflection error $\triangle F l / F l$ during 350 nsec after the source reached nominal value for periodic and matched kicker. $\nu_{\text {max }}=120 \mathrm{MHz}$.

Time interval between successive points of curve: $\tau=4.17$ nsec.

Note reduction in magnitude of the errors and in settling time, as compared to fig. 7 


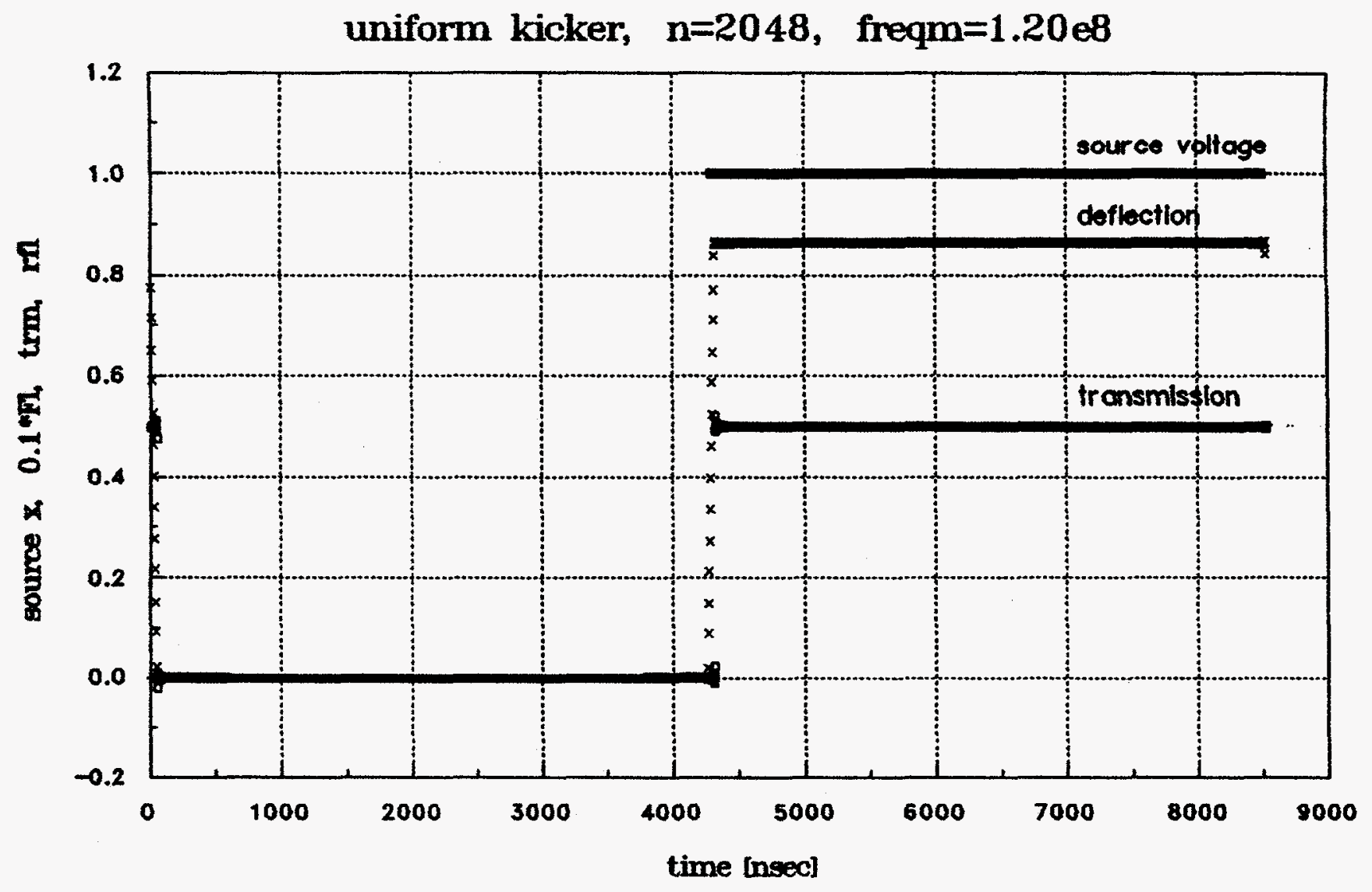

Fig. 14. Source voltage, deflection factor FI, transmission factor trm and reflection factor rfl as functions of time for longitudinally uniform and matched kicker. 'Square' wave excitation with period 8.533 usec, $\nu_{\max }=120 \mathrm{MHz}$. 
uniform kicker, $n=2048$, freqm $=1.20 \mathrm{e8}$

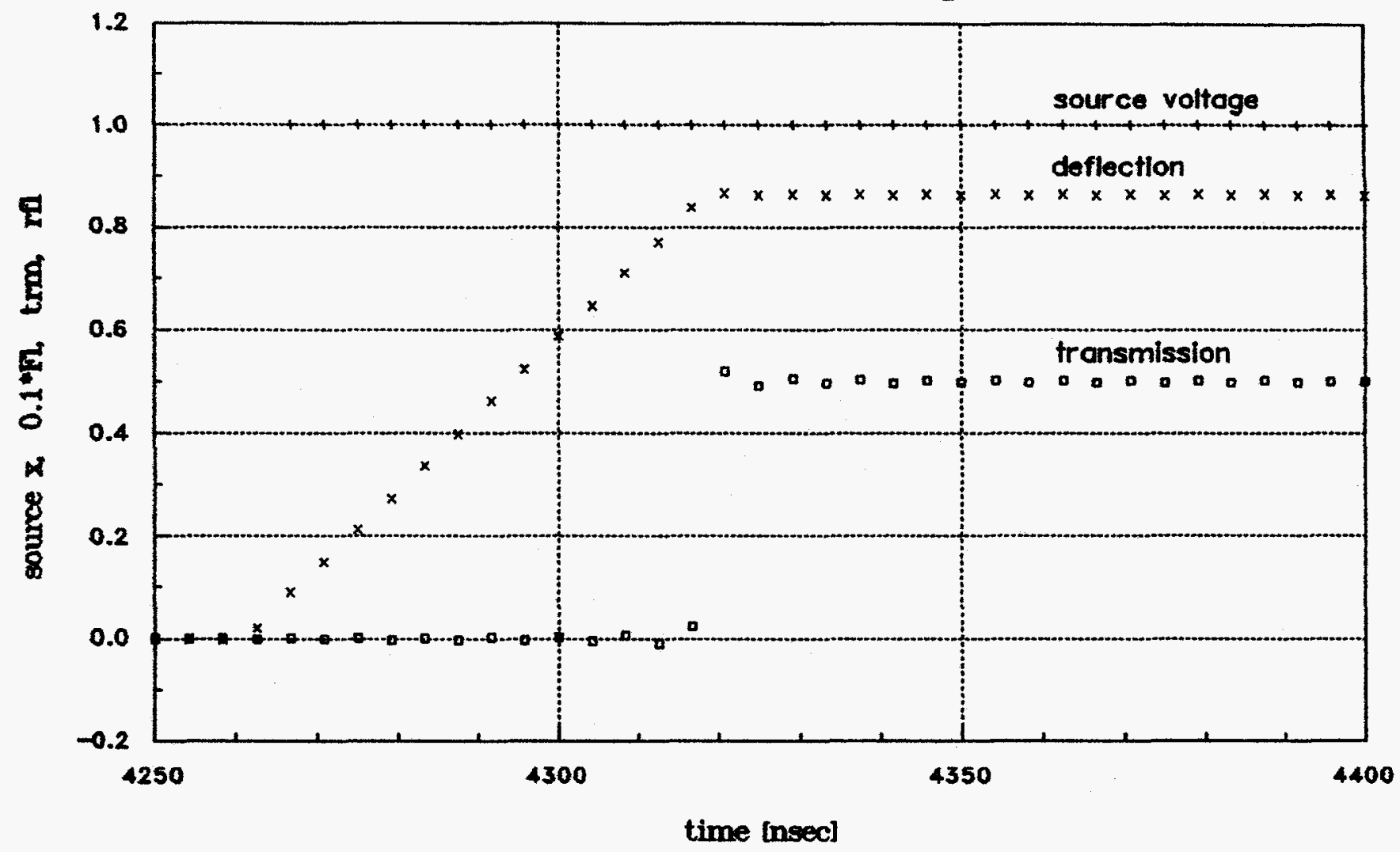

Fig. 15. Source voltage, deflection factor Fl, transmission factor trm and as functions of time for longitudinally uniform and matched kicker.

'Square' wave excitation with period $8.533 \mathrm{\mu sec}$, $\nu_{\max }=120 \mathrm{MHz}$. Time interval shown $4.25 \mu \mathrm{sec} \leq \mathrm{t} \leq 4.4 \mu \mathrm{sec}$. Time interval between successive points of each curve: 4.17 nsec Note reduction of irregularities in F1, trm as compared to figs 6 and 12 . 


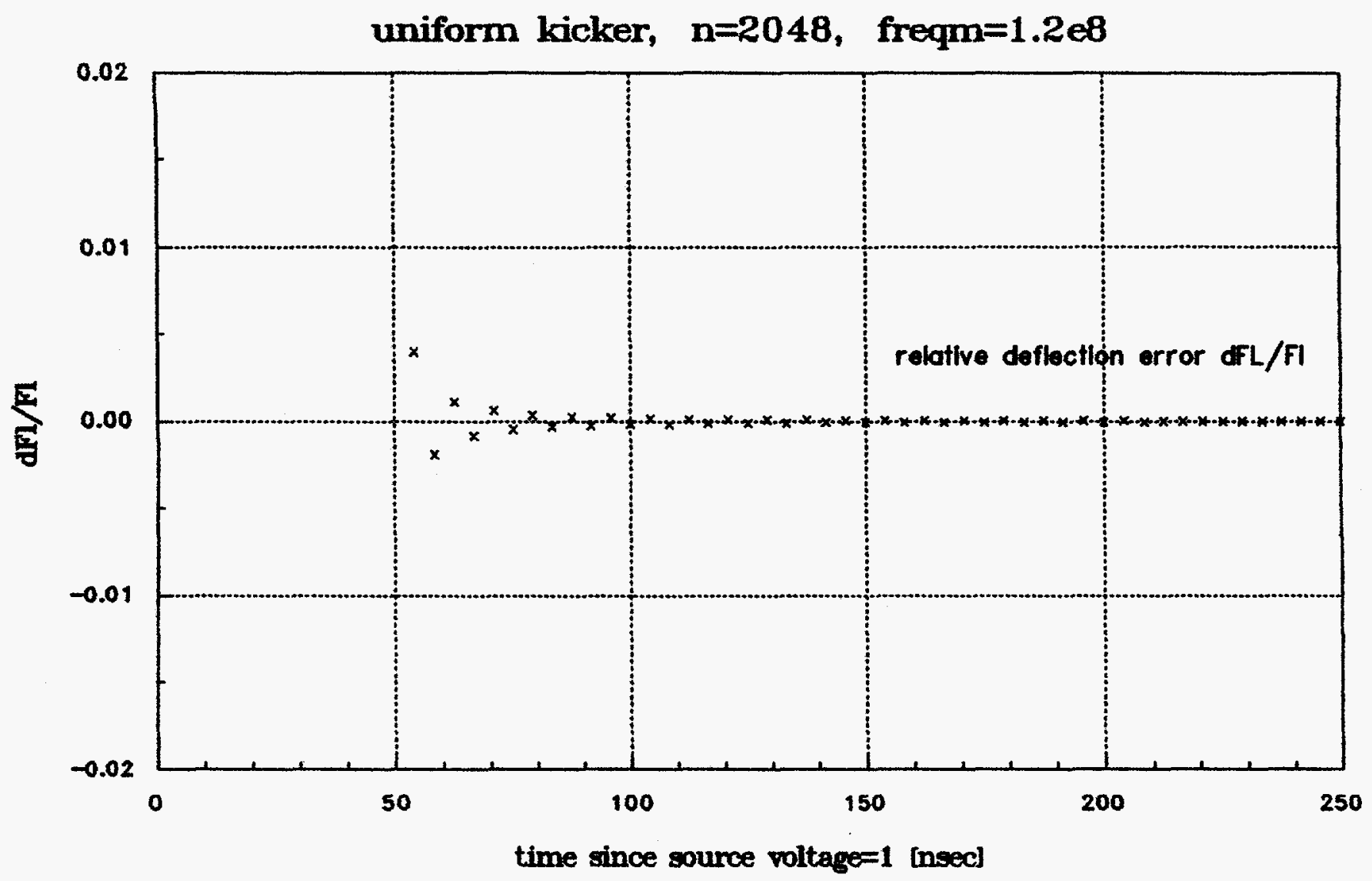

Fig. 16. Relative deflection error $\triangle F l / F 1$ during 250 nsec after the source voltage reached nominal value for longitudinally uniform and matched kicker. $\nu_{\max }=120 \mathrm{MHz}$. Time interval between successive points of curve: $\tau=4.17 \mathrm{nsec}$.

Note further reduction in magnitude of the errors and in settling time as compared with figs. 7 and 13 . 


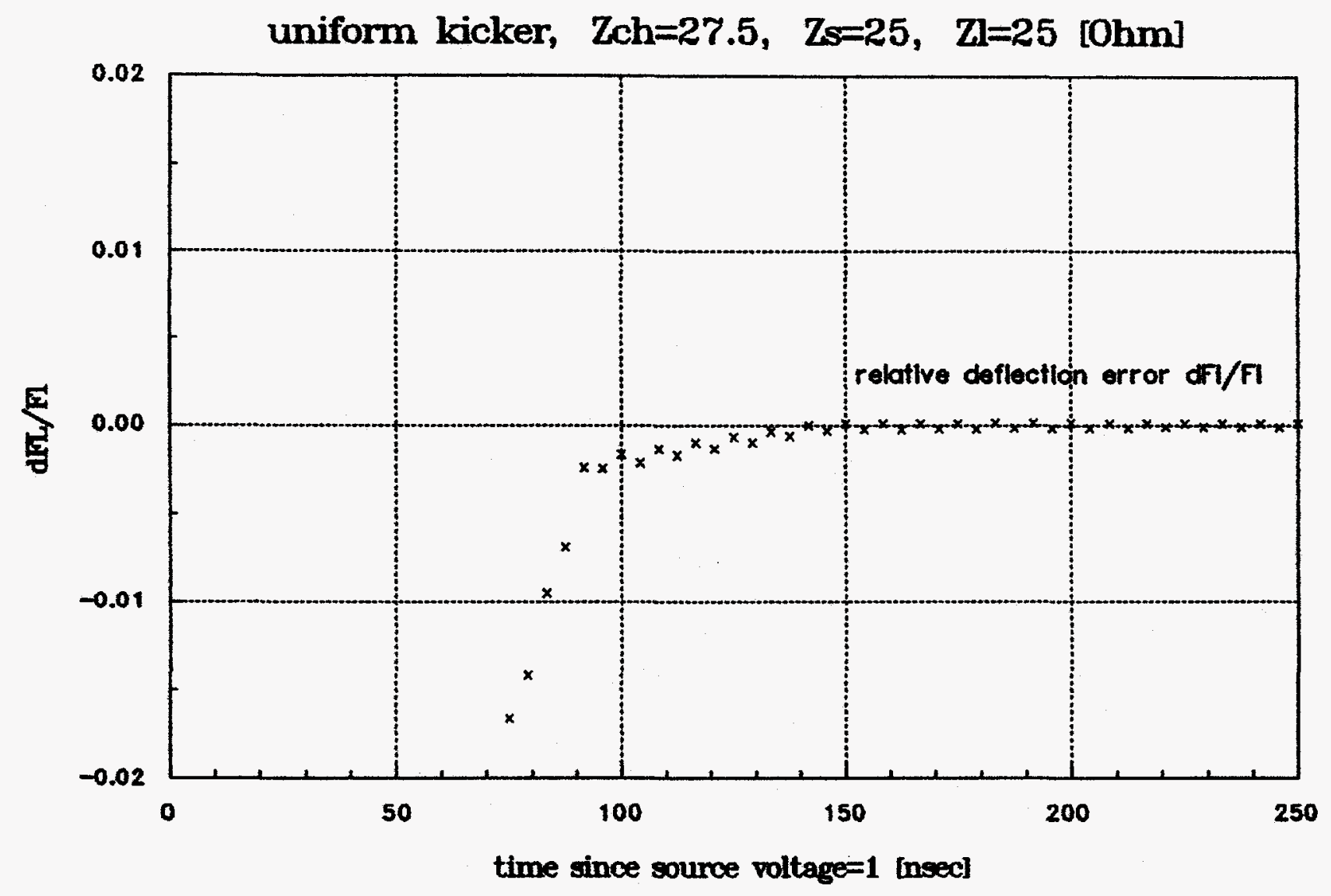

Fig. 17. Consequence of a mismatch between kicker impedance and source impedance for the relative deflection error of a longitudinally uniform kicker.

Note that the instant from which the error is acceptably small has shifted to later times as compared with fig. 16 . 


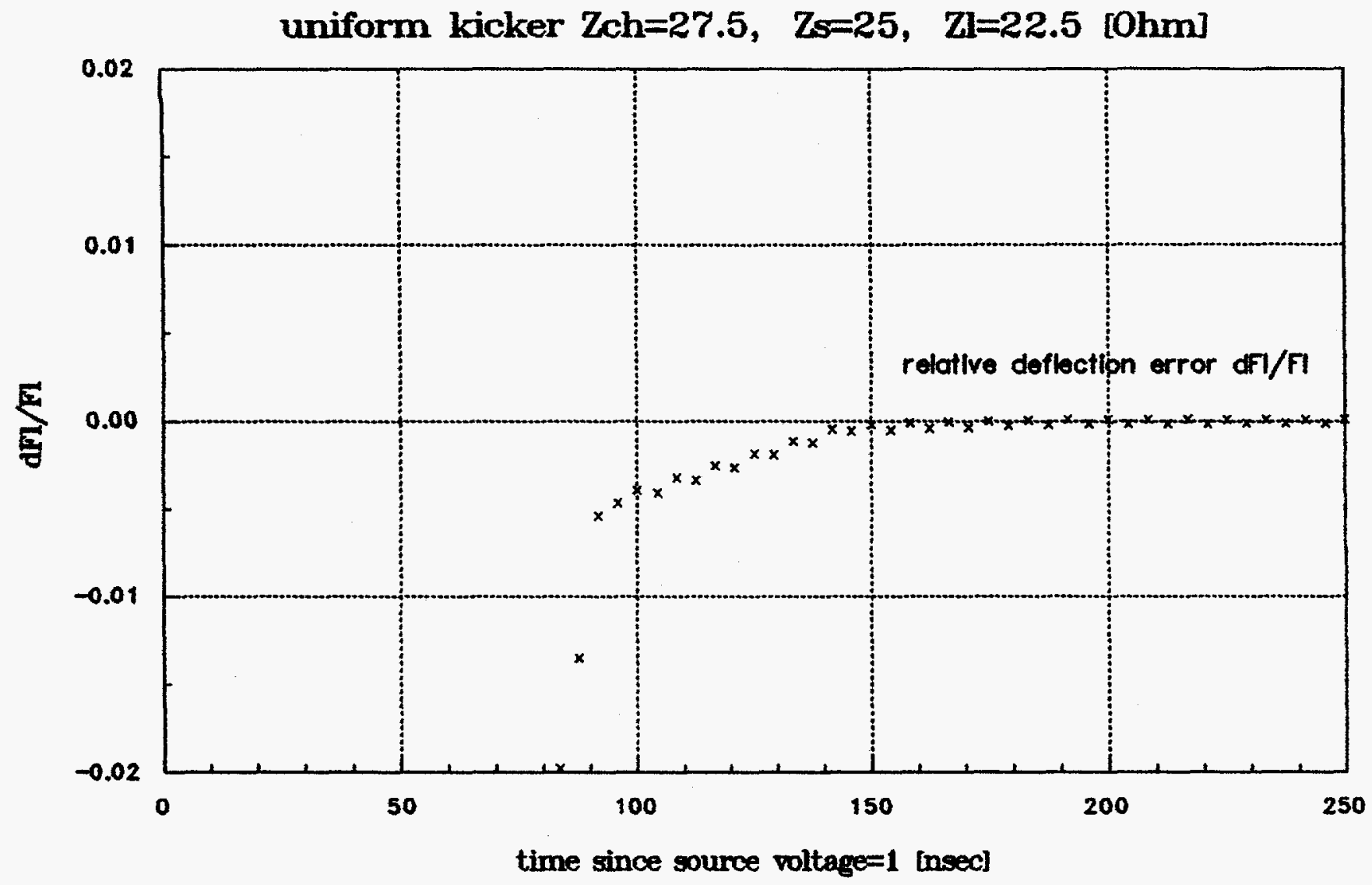

Fig. 18. Consequence of mismatches between the impedances of kicker, source and load for the relative deflection error of a longitudinally uniform kicker.

Note that the error has become larger, but that the shift in time remained about the same as in fig. 17. 